\title{
Some Integral Inequalities for Generalized Convex Fuzzy-Interval-Valued Functions via Fuzzy Riemann Integrals
}

\author{
Muhammad Bilal Khan ${ }^{1} \cdot$ Muhammad Aslam Noor ${ }^{1}$ Pshtiwan Othman Mohammed ${ }^{2}$ Juan L. G. Guirao ${ }^{3,4}$. \\ Khalida Inayat Noor ${ }^{1}$
}

Received: 23 March 2021 / Accepted: 19 August 2021

(c) The Author(s) 2021

\begin{abstract}
In this study, we introduce the new concept of $h$-convex fuzzy-interval-valued functions. Under the new concept, we present new versions of Hermite-Hadamard inequalities (H-H inequalities) are called fuzzy-interval Hermite-Hadamard type inequalities for $h$-convex fuzzy-interval-valued functions ( $h$-convex FIVF) by means of fuzzy order relation. This fuzzy order relation is defined level wise through Kulisch-Miranker order relation defined on fuzzy-interval space. Fuzzy order relation and inclusion relation are two different concepts. With the help of fuzzy order relation, we also present some $\mathrm{H}-\mathrm{H}$ type inequalities for the product of $h$-convex FIVFs. Moreover, we have also established strong relationship between Hermite-Hadamard-Fej'er (H-H-Fej'er) type inequality and $h$-convex FIVF. There are also some special cases presented that can be considered applications. There are useful examples provided to demonstrate the applicability of the concepts proposed in this study. This paper's thoughts and methodologies could serve as a springboard for more research in this field.
\end{abstract}

Keywords Convex fuzzy-interval-valued function $\cdot$ Fuzzy Riemann integral $\cdot$ Fuzzy-interval Hermite $\cdot$ Hadamard inequality $\cdot$ Fuzzy-interval Hermite $\cdot$ Hadamard $\cdot$ Fej'er inequality

\section{Introduction}

Muhammad Bilal Khan

bilal42742@gmail.com

Muhammad Aslam Noor

noormaslam@gmail.com

Pshtiwan Othman Mohammed

pshtiwansangawi@gmail.com

Juan L. G. Guirao

juan.garcia@upct.es

Khalida Inayat Noor

khalidanoor@hotmail.com

1 Department of Mathematics, COMSATS University Islamabad, Islamabad, Pakistan

2 Department of Mathematics, College of Education, University of Sulaimani, Sulaimani, Kurdistan Region, Iraq

3 Department of Applied Mathematics and Statistics, Technical University of Cartagena, Hospital de Marina, 30203 Cartagena, Spain

4 Nonlinear Analysis and Applied Mathematics (NAAM)-Research Group, Department of Mathematics, Faculty of Science, King Abdulaziz University, P.O. Box 80203, Jidda 21589, Saudi Arabia
The significance and supreme applications of convex functions are well known in different fields, especially in the study of integral inequalities, variational inequalities and optimization. Therefore, much attention has been given in studying and characterizing different directions of classical idea of convexity. Recently, many extensions and generalizations of convex functions have been studied. For more useful details, see $[1-4,6,7,14,18-21,25]$ and the references are therein. In classical approach, a real valued function $\Psi: K \rightarrow \mathbb{R}$ is called convex if

$\Psi(\xi z+(1-\xi) y) \leq \xi \Psi(z)+(1-\xi) \Psi(y)$,

for all $z, \epsilon y \in K, \xi \in[0,1]$.

The concept of convexity with integral problem is an interesting area for research. Therefore, many inequalities have been introduced as applications of convex functions. Among those, the $\mathrm{H}-\mathrm{H}$ inequality is an interesting outcome in convex analysis. The $\mathrm{H}-\mathrm{H}$ inequality $[16,17]$ for convex function $\Psi: K \rightarrow \mathbb{R}$ on an interval $K=[u, v]$ 
$\Psi\left(\frac{u+v}{2}\right) \leq \frac{1}{v-u} \int_{u}^{v} \Psi(z) \mathrm{d} z \leq \frac{\Psi(u)+\Psi(v)}{2}$,

for all $z \in K$.

On the other hand, the concept of interval analysis was proposed and investigated by Moore [24], and Kulish and Miranker [23] to improve the reliability of the calculation results and automatically perform error analysis. It is a discipline in which an uncertain variable is represented by an interval of real numbers and real operations are replaced by interval operation. In last 5 decades, the concept of interval was used as a tool to handle uncertain problems. Recently, many authors have contributed their role in this theory and introduced new concepts. Zhao et al. [30] introduced $h$-convex interval-valued functions and proved that the following $\mathrm{H}-\mathrm{H}$ type inequality for $h$-convex intervalvalued functions:

Theorem 1.1. Let $\Psi:[u, v] \subset \mathbb{R} \rightarrow \mathcal{K}_{C}^{+}$be a h-convex interval-valued function given by $\Psi(z)=\left[\Psi_{*}(z), \Psi^{*}(z)\right]$ for all $z \in[u, v]$, where $\Psi_{*}(z)$ is a h-convex function and $\Psi^{*}(z)$ is a h-concave function. If $\Psi$ is Riemann integrable, then

$\frac{1}{2 h\left(\frac{1}{2}\right)} \Psi\left(\frac{u+v}{2}\right) \supseteq \frac{1}{v-u} \int_{u}^{v} \Psi(z) \mathrm{d} z$

$\supseteq \frac{\Psi(u)+\Psi(v)}{2} \int_{0}^{1} h(\xi) \mathrm{d} \xi$.

If $\Psi_{*}(z),=\Psi^{*}(z)$, then integral inequality (3) reduces for $h$ -convex function, see [29]. We refer to the readers for further analysis of literature on the applications and properties of generalized convex functions and $\mathrm{H}-\mathrm{H}$ integral inequalities, see $[5,8,9,15,17,22,27,28,31-48]$ and the references therein.

There are some integrals to deal with fuzzy-intervalvalued functions (FIVF), where the integrands are FIVFs. For instance, Oseuna-Gomez et al. [26], and Costa [11] constructed $\mathrm{H}-\mathrm{H}$ and Jensen's integral inequality for FIVFs using fuzzy Aumann integrals. Using same approach Costa and Floures [10] and Costa et al. [12] also presented Minkowski and Beckenbach's inequalities, where the integrands are interval-valued and FIVFs. Motivated by $[10,11$, $26,29]$ and [30], we generalize integral inequality (2) by constructing fuzzy-interval integral inequality for $h$-convex FIVF, where the integrands are $h$-convex FIVFs.

This study is organized as follows: Sect. 2 presents preliminary notions, new concepts and results in interval space, in the space of fuzzy-intervals and for $h$-convex FIVFs. Section 3 obtains fuzzy-interval $\mathrm{H}-\mathrm{H}$ inequalities via $h$-convex FIVFs. In addition, some interesting examples are also given to verify our results. Section 4 gives conclusions.

\section{Preliminaries}

In this section, we recall some basic preliminary notions, definitions and results. With the help of these results, some new basic definitions and results are also discussed.

We begin by recalling the basic notations and definitions. We define interval as, $\left[\omega_{*}, \omega^{*}\right]=\left\{z \in \mathbb{R}: \omega_{*} \leq z \leq \omega^{*}\right.$ and $\left.\omega_{*}, \omega^{*} \in \mathbb{R}\right\}$, where $\omega_{*} \leq \omega^{*}$.

We write len $\left[\omega_{*}, \omega^{*}\right]=\omega^{*}-\omega_{*}$, If len $\left[\omega_{*}, \omega^{*}\right]=0$ then, $\left[\omega_{*}, \omega^{*}\right]$ is called degenerate. In this article, all intervals will be non-degenerate intervals. The collection of all closed and bounded intervals of $\mathbb{R}$ is denoted and defined as $\mathcal{K}_{C}=\left\{\left[\omega_{*}, \omega^{*}\right]: \omega_{*}, \omega^{*} \in \mathbb{R a n d} \omega_{*} \leq \omega^{*}\right\}$. If $\omega_{*} \geq 0$ then, $\left[\omega_{*}, \omega^{*}\right]$ is called positive interval. The set of all positive interval is denoted by $\mathcal{K}_{C}^{+}$and defined as $\mathcal{K}_{C}^{+}=\left\{\left[\omega_{*}, \omega^{*}\right]:\left[\omega_{*}, \omega^{*}\right] \in \mathcal{K}_{C}\right.$ and $\left.\omega_{*} \geq 0\right\}$.

We'll now look at some of the properties of intervals using arithmetic operations. Let $\left[\varrho_{*}, \varrho^{*}\right],\left[\&_{*}, \&^{*}\right] \in \mathcal{K}_{C}$ and $\rho \in \mathbb{R}$, then we have

$\left[\varrho_{*}, \varrho^{*}\right]+\left[\&_{*}, \&^{*}\right]=\left[\varrho_{*}+\&_{*}, \varrho^{*}+\&^{*}\right]$,

$\left[\varrho_{*}, \varrho^{*}\right] \times\left[\&_{*}, \&^{*}\right]=\left[\begin{array}{l}\min \left\{\varrho_{*} \&_{*}, \varrho^{*} \&_{*}, \varrho_{*} \&^{*}, \varrho^{*} \&^{*}\right\} \\ \max \left\{\varrho_{*} \&_{*}, \varrho^{*} \&_{*}, \varrho_{*} \&^{*}, \varrho^{*} \&^{*}\right\}\end{array}\right]$

$\rho .\left[\varrho_{*}, \varrho^{*}\right]=\left\{\begin{array}{l}{\left[\rho \varrho_{*}, \rho \varrho^{*}\right] \text { if } \rho \geq 0,} \\ \left.\rho \varrho^{*}, \rho \varrho_{*}\right] \text { if } \rho<0 .\end{array}\right.$

For $\left[\varrho_{*}, \varrho^{*}\right],\left[\&_{*}, \&^{*}\right] \in \mathcal{K}_{C}$, the inclusion " $\subseteq$ " is defined by $\left[\varrho_{*}, \varrho^{*}\right] \subseteq\left[\&_{*}, \&^{*}\right]$, if and only if $\&_{*} \leq \varrho_{*}, \varrho^{*} \leq \&^{*}$.

Remark 2.1. The relation " $\leq_{I}$ " defined on $\mathcal{K}_{C}$ by $\left[\varrho_{*}, \varrho^{*}\right] \leq_{I}\left[\&_{*}, \&^{*}\right]$ if and only if $\varrho_{*} \leq \&_{*}, \varrho^{*} \leq \&^{*}$, for all $\left[\varrho_{*}, \varrho^{*}\right],\left[\&_{*}, \&^{*}\right] \in \mathcal{K}_{C}$, it is an order relation, see [23]. For given $\left[\varrho_{*}, \varrho^{*}\right],\left[\&_{*}, \&^{*}\right] \in \mathcal{K}_{C}$, we say that $\left[\varrho_{*}, \varrho^{*}\right] \leq_{I}\left[\&_{*}, \&^{*}\right]$ if and only if $\varrho_{*} \leq \&_{*}, \varrho^{*} \leq \&^{*}$ or $\varrho_{*} \leq \&_{*}, \varrho^{*}<\&^{*}$.

Moore [24] initially proposed the concept of Riemann integral for IVF, which is defined as follows:

Theorem 2.2. [24] If $\Psi:[u, v] \subset \mathbb{R} \rightarrow \mathcal{K}_{C}$ is an IVF on such that $\Psi(z)=\left[\Psi_{*}, \Psi^{*}\right]$. Then $\Psi$ is Riemann integrable over $[u, v]$ if and only if, $\Psi_{*}$ and $\Psi^{*}$ both are Riemann integrable over $[u, v]$ such that 


$$
\begin{aligned}
& \text { (IR) } \int_{u}^{v} \Psi(z) \mathrm{d} z \\
& =\left[(R) \int_{u}^{v} \Psi_{*}(z) \mathrm{d} z,(R) \int_{u}^{v} \Psi^{*}(z) \mathrm{d} z\right]
\end{aligned}
$$

Let $\mathbb{R}$ be the set of real numbers. A mapping $\zeta: \mathbb{R} \rightarrow[0,1]$ called the membership function distinguishes a fuzzy subset set $\mathcal{A}$ of $\mathbb{R}$. This representation is found to be acceptable in this study. $\mathbb{F}(\mathbb{R})$ also stand for the collection of all fuzzy subsets of $\mathbb{R}$.

A real fuzzy interval $\zeta$ is a fuzzy set in $\mathbb{R}$ with the following properties:

(1) $\zeta$ is normal i.e. there exists $z \in \mathbb{R}$ such that $\zeta(z)=1$;

(2) $\zeta$ is upper semi continuous i.e., for given $z \in \mathbb{R}$, for every $z \in \mathbb{R}$ there exist $\varepsilon>0$ there exist $\delta>0$ such that $\zeta(z)-\zeta(y)<\varepsilon$ for all $y \in \mathbb{R}$ with $|z-y|<\delta$.

(3) $\zeta$ is fuzzy convex i.e., $\zeta((1-\xi) z+\xi y) \geq \min (\zeta(z), \zeta(y))$, $\forall z, y \in \mathbb{R}$ and $\xi \in[0,1]$

(4) $\zeta$ is compactly supported i.e., $\operatorname{cl}\{z \in \mathbb{R}|\zeta(z)\rangle 0\}$ is compact.

The collection of all real fuzzy intervals is denoted by $\mathbb{F}_{0}$.

Let $\zeta \in \mathbb{F}_{0}$ be real fuzzy interval, if and only if, $\beta$-levels $[\zeta]^{\beta}$ is a nonempty compact convex set of $\mathbb{R}$. This is represented by

$[\zeta]^{\beta}=\{z \in \mathbb{R} \mid \zeta(z) \geq \beta\}$,

from these definitions, we have

$[\zeta]^{\beta}=\left[\zeta_{*}(\beta), \zeta^{*}(\beta)\right]$

where

$\zeta_{*}(\beta)=\inf \{z \in \mathbb{R} \mid \zeta(z) \geq \beta\}$,

$\zeta^{*}(\beta)=\sup \{z \in \mathbb{R} \mid \zeta(z) \geq \beta\}$

Thus a real fuzzy interval $\zeta$ can be identified by a parametrized triples

$\left\{\left(\zeta_{*}(\beta), \zeta^{*}(\beta), \beta\right): \beta \in[0,1]\right\}$.

These two end point functions $\zeta_{*}(\beta)$ and $\zeta^{*}(\beta)$ are used to characterize a real fuzzy interval as a result.

Proposition 2.3. [10] Let $\zeta, \Theta \in \mathbb{F}_{0}$. Then fuzzy order relation " $\leqslant$ " given on $\mathbb{F}_{0}$ by

$\zeta \preccurlyeq \Theta$ if and only if, $[\zeta]^{\beta} \leq_{I}[\Theta]^{\beta}$ for all $\beta \in(0,1]$, it is partial order relation.

We will now look at some of the properties of fuzzy intervals using arithmetic operations. Let $\zeta, \Theta \in \mathbb{F}_{0}$ and $\rho \in \mathbb{R}$, then we have

$[\zeta \tilde{+} \Theta]^{\beta}=[\zeta]^{\beta}+[\Theta]^{\beta}$,

$[\zeta \tilde{\times} \Theta]^{\beta}=[\zeta]^{\beta} \times[\Theta]^{\beta}$,

$[\rho . \zeta]^{\beta}=\rho \cdot[\zeta]^{\beta}$.

For $\psi \in \mathbb{F}_{0}$ such that $\zeta=\Theta \tilde{+} \psi$, we have the existence of the Hukuhara difference of $\zeta$ and $\Theta$, which we call the $\mathrm{H}$-difference of $\zeta$ and $\Theta$, and denoted by $\zeta \simeq \Theta$. If H-difference exists, then

$(\psi)^{*}(\beta)=(\zeta \simeq \Theta)^{*}(\beta)=\zeta^{*}(\beta)-\Theta^{*}(\beta)$

$(\psi)_{*}(\beta)=(\zeta \simeq \Theta)_{*}(\beta)=\zeta_{*}(\beta)-\Theta_{*}(\beta)$.

Theorem 2.4. $[13,26]$ The space $\mathbb{F}_{0}$ dealing with a supremum metric i.e., for $\psi, \Theta \in \mathbb{F}_{0}$

$D(\psi, \Theta)=\sup _{0 \leq \beta \leq 1} H\left([\zeta]^{\beta},[\Theta]^{\beta}\right)$,

it is a complete metric space, where $H$ denote the wellknown Hausdorff metric on space of intervals.

Definition 2.5. [10] A fuzzy-interval-valued map $\Psi: K \subset \mathbb{R} \rightarrow \mathbb{F}_{0}$ is called FIVF. For each $\beta \in(0,1]$, whose $\beta$-levels define the family of IVFs $\Psi_{\beta}: K \subset \mathbb{R} \rightarrow \mathcal{K}_{C}$ are given by $\Psi_{\beta}(z)=\left[\Psi_{*}(z, \beta), \Psi^{*}(z, \beta)\right]$ for all $z \in K$. Here, for each $\beta \in(0,1]$, the end point real functions $\Psi_{*}(., \beta), \Psi^{*}(., \beta): K \rightarrow \mathbb{R}$ are called lower and upper functions of $\Psi$.

The following conclusions can be drawn from the preceding literature review [10, 13, 22, 24]:

Definition 2.6. Let $\Psi:[u, v] \subset \mathbb{R} \rightarrow \mathbb{F}_{0}$ be a FIVF. Then fuzzy integral of $\Psi$ over $[u, v]$, denoted by $(\mathrm{FR}) \int_{u}^{v} \Psi(z) \mathrm{d} z$, it is given level-wise by

$\left[(\mathrm{FR}) \int_{u}^{v} \Psi(z) \mathrm{d} z\right]^{\beta}=(\mathrm{IR}) \int_{u}^{v} \Psi_{\beta}(z) \mathrm{d} z$ 


$$
=\left\{\int_{u}^{v} \Psi(z, \beta) \mathrm{d} z: \Psi(z, \beta) \in \mathcal{R}_{([u, v], \beta)}\right\},
$$

for all $\beta \in(0,1]$, where $\mathcal{R}_{([u, v], \beta)}$ denotes the collection of Riemannian integrable functions of IVFs. $\Psi$ is $F R$-integrable over $[u, v]$ if $(\mathrm{FR}) \int_{u} \Psi(z) \mathrm{d} z \in \mathbb{F}_{0}$. Note that, if both end point functions are Lebesgue-integrable, then $\Psi$ is fuzzy Aumannintegrable function over $[u, v]$, see $[13,22,24]$.

Theorem 2.7. Let $\Psi:[u, v] \subset \mathbb{R} \rightarrow \mathbb{F}_{0}$ be a FIVF, whose $\beta$ -levels define the family of IVFs $\Psi_{\beta}:[u, v] \subset \mathbb{R} \rightarrow \mathcal{K}_{C}$ are given by $\Psi_{\beta}(z)=\left[\Psi_{*}(z, \beta), \Psi^{*}(z, \beta)\right]$ for all $z \in[u, v]$ and for all $\beta \in(0,1]$. Then $\Psi$ is FR-integrable over $[u, v]$ if and only if, $\Psi_{*}(z, \beta)$ and $\Psi^{*}(z, \beta)$ both are R-integrable over $[u, v]$. Moreover, if $\Psi$ is FR-integrable over $[u, v]$, then.

$$
\begin{aligned}
& {\left[(\mathrm{FR}) \int_{u}^{v} \Psi(z) \mathrm{d} z\right]^{\beta}} \\
& =\left[(R) \int_{u}^{v} \Psi_{*}(z, \beta) \mathrm{d} z,(R) \int_{u}^{v} \Psi^{*}(z, \beta) \mathrm{d} z\right] \\
& =(\mathrm{IR}) \int_{u}^{v} \Psi_{\beta}(z) \mathrm{d} z
\end{aligned}
$$

for all $\beta \in(0,1]$. For all $\beta \in(0,1], \mathcal{F R}_{([\mu, \nu], \beta)}$ denotes the collection of all FR-integrable FIVFs over $[\mu, \nu]$.

Definition 2.8. Let $K$ be convex set and $h:[0,1] \subseteq K \rightarrow \mathbb{R}$ such that $h \equiv 0$. Then FIVF $\Psi: K \rightarrow \mathbb{F}_{C}(\mathbb{R})$ is said to be:

- $h$-convex on $K$ if

$\Psi(\xi z+(1-\xi) y) \preccurlyeq h(\xi) \Psi(z) \tilde{+} h(1-\xi) \Psi(y)$,

for all $z, y \in K, \xi \in[0,1]$, where $\Psi(z) \geqslant \tilde{0}$.

- $h$-concave on $K$ if inequality (11) is reversed.

- affine $h$-convex on $K$ if

$$
\Psi(\xi z+(1-\xi) y)=h(\xi) \Psi(z) \tilde{+} h(1-\xi) \Psi(y),
$$

for all $z, y \in K, \xi \in[0,1]$, where $\Psi(z) \geqslant \tilde{0}$.

Remark 2.9. The $h$-convex FIVFs have some very nice properties similar to convex FIVF,

If $\Psi$ is $h$-convex FIVF, then $\Upsilon \Psi$ is also $h$-convex for $\Upsilon \geq 0$

If $\Psi$ and $\Psi$ both are $h$-convex FIVFs, then $\max (\Psi(z), \Psi(z))$ is also $h$-convex FIVF.
We now discuss some special cases of $h$-convex FIVFs:

(i) If $h(\xi)=\xi^{s}$, then $h$-convex FIVF becomes $s$-convex FIVF, that is

$\Psi(\xi z+(1-\xi) y) \preccurlyeq \xi^{s} \Psi(z) \tilde{+}(1-\xi)^{s} \Psi(y)$,

for all $z, y \in K, \xi \in[0,1]$.

(ii) If $h(\xi)=\xi$, then $h$-convex FIVF becomes convex FIVF, see [25], that is

$\Psi(\xi z+(1-\xi) y) \leqslant \xi \Psi(z) \tilde{+}(1-\xi) \Psi(y)$,

for all $z, y \in K, \xi \in[0,1]$.

(iii) If $h(\xi) \equiv 1$, then $h$-convex FIVF becomes $P$-convex FIVF, that is

$\Psi(\xi z+(1-\xi) y) \preccurlyeq \Psi(z) \tilde{+} \Psi(y)$,

for all $z, y \in K, \xi \in[0,1]$.

Note that, special cases (i) and (iii) are also new ones.

Theorem 2.10. Let $K$ be convex set, non-negative real valued function $h:[0,1] \subseteq K \rightarrow \mathbb{R}$ such that $h \equiv 0$ and let $\Psi: K \rightarrow \mathbb{F}_{C}(\mathbb{R})$ be a FIVF, whose $\beta$-levels define the family of interval valued functions $\Psi_{\beta}: K \subset \mathbb{R} \rightarrow \mathcal{K}_{C}^{+} \subset \mathcal{K}_{C}$ are given by

$\Psi_{\beta}(z)=\left[\Psi_{*}(z, \beta), \Psi^{*}(z, \beta)\right]$,

for all $z \in K$ and for all $\beta \in[0,1]$. Then $\Psi$ is $h$-convex on $K$, if and only if, for all $\beta \in[0,1], \Psi_{*}(z, \beta)$ and $\Psi^{*}(z, \beta)$ are h-convex.

Proof. Assume that for each $\beta \in[0,1], \Psi_{*}(z, \beta)$ and $\Psi^{*}(z, \beta)$ are $h$-convex on $K$. Then from (11), we have.

$\Psi_{*}(\xi z+(1-\xi) y, \beta)$

$\leq h(\xi) \Psi_{*}(z, \beta)+h(1-\xi) \Psi_{*}(y, \beta)$

for all $z, y \in K, \xi \in[0,1]$,and

$\Psi^{*}(\xi z+(1-\xi) y, \beta)$

$\leq h(\xi) \Psi^{*}(z, \beta)+h(1-\xi) \Psi^{*}(y, \beta)$,

for all $z, y \in K, \xi \in[0,1]$.

Then by (13), (4) and (6), we obtain

$\Psi_{\beta}(\xi z+(1-\xi) y)$

$=\left[\Psi_{*}(\xi z+(1-\xi) y, \beta), \Psi^{*}(\xi z+(1-\xi) y, \beta)\right]$,

$\leq_{I}\left[h(\xi) \Psi_{*}(z, \beta), h(\xi) \Psi^{*}(z, \beta)\right]$ 
$+\left[h(1-\xi) \Psi_{*}(y, \beta), h(1-\xi) \Psi^{*}(y, \beta)\right]$,

that is

$\Psi(\xi z+(1-\xi) y) \leqslant h(\xi) \Psi(z) \tilde{+} h(1-\xi) \Psi(y), \forall z, y \in K, \xi \in[0,1]$.

Hence, $\Psi$ is $h$-convex FIVF on $K$.

Conversely, let $\Psi$ is $h$-convex FIVF on $K$. Then for all $z, y \in K$ and $\xi \in[0,1]$, we have

$\Psi(\xi z+(1-\xi) y) \leqslant h(\xi) \Psi(z) \tilde{+} h(1-\xi) \Psi(y)$.

Therefore, from (13), we have

$\Psi_{\beta}(\xi z+(1-\xi) y)$

$=\left[\Psi_{*}(\xi z+(1-\xi) y, \beta), \Psi^{*}(\xi z+(1-\xi) y, \beta)\right]$.

Again, from (13), we obtain

$h(\xi) \Psi_{\beta}(z)+h(1-\xi) \Psi_{\beta}(z)$

$=\left[h(\xi) \Psi_{*}(z, \beta), h(\xi) \Psi^{*}(z, \beta)\right]$

$+\left[h(1-\xi) \Psi_{*}(y, \beta), h(1-\xi) \Psi^{*}(y, \beta)\right]$,

for all $z, y \in K$ and $\xi \in[0,1]$. Then by $h$-convexity of $\Psi$, we have for all $z, y \in K$ and $\xi \in[0,1]$ such that

$\Psi_{*}(\xi z+(1-\xi) y, \beta) \leq h(\xi) \Psi_{*}(z, \beta)+h(1-\xi) \Psi_{*}(y, \beta)$,

and

$\Psi^{*}(\xi z+(1-\xi) y, \beta)$

$\leq h(\xi) \Psi^{*}(z, \beta)+h(1-\xi) \Psi^{*}(y, \beta)$,

for each $\beta \in[0,1]$. Hence, this concludes the proof.

Remark 2.11. If $\Psi_{*}(z, \beta)=\Psi^{*}(z, \beta)$ with $\beta=1$, then $h$-convex FIVF reduces to the classical $h$-convex function, see [29]. If $\Psi_{*}(z, \beta)=\Psi^{*}(z, \beta)$ with $\beta=1$ and $h(\xi)=\xi^{s}$ with $s \in(0,1)$ then $h$-convex FIVF becomes the classical $s$-convex function, see [6].

If $\Psi_{*}(z, \beta)=\Psi^{*}(z, \beta)$ with $\beta=1$ and $h(\xi)=\xi$, then $h$-convex FIVF reduces to the classical convex function.

If $\Psi_{*}(z, \beta)=\Psi^{*}(z, \beta)$ with $\beta=1$ and $h(\xi)=1$ then $h$-convex FIVF becomes the classical $p$-convex function, see [14].

Example 2.12. We consider $h(\xi)=\xi$, for $\xi \in[0,1]$ and the FIVF $\Psi:[0,1] \rightarrow \mathbb{F}_{C}(\mathbb{R})$ defined by
$\Psi(z)(\sigma)=\left\{\begin{array}{c}\frac{\sigma}{2 z^{2}} \sigma \in\left[0,2 z^{2}\right] \\ \frac{4 z^{2}-\sigma}{2 z^{2}} \sigma \in\left(2 z^{2}, 4 z^{2}\right] \\ \text { Ootherwise, }\end{array}\right.$

Then, for each $\beta \in[0,1]$, we have $\Psi_{\beta}(z)=\left[2 \beta z^{2},(4-2 \beta) z^{2}\right]$. Since end point functions $\Psi_{*}(z, \beta), \Psi^{*}(z, \beta)$ are $h$-convex functions for each $\beta \in[0,1]$. Hence $\Psi(z)$ is $h$-convex FIVF.

\section{Main Results}

In this section, we propose fuzzy-interval $\mathrm{H}-\mathrm{H}$ inequalities for $h$-convex FIVFs. Furthermore, several examples are given to demonstrate the applicability of the theory produced in this research.

Theorem 3.1. Let $\Psi:[\sqcap, \nu] \rightarrow \mathbb{F}_{C}(\mathbb{R})$ be a h-convex FIVF with non-negative real valued function $h:[0,1] \rightarrow \mathbb{R}$ and $h\left(\frac{1}{2}\right) \neq 0$, and for all $\beta \in[0,1], \Psi_{\beta}: K \subset \mathbb{R} \rightarrow \mathcal{K}_{C}{ }^{+} \subset \mathcal{K}_{C}$ represent the family of IVFs through $\beta$-levels. If $\Psi \in \mathcal{F R}_{([\sqcap, v], \beta)}$, then

$\frac{1}{2 h\left(\frac{1}{2}\right)} \Psi\left(\frac{\sqcap+v}{2}\right) \leqslant \frac{1}{v-\sqcap}(F R) \int_{\Pi}^{v} \Psi(\ddagger) \mathrm{d} \ddagger$

$\preccurlyeq[\Psi(\sqcap) \tilde{+} \Psi(v)] \int_{0}^{1} h(\xi) \mathrm{d} \xi$.

Proof. Let $\Psi:[\sqcap, \nu] \rightarrow \mathbb{F}_{C}(\mathbb{R}), h$-convex FIVF. Then, by hypothesis, we have

$\frac{1}{h\left(\frac{1}{2}\right)} \Psi\left(\frac{\sqcap+v}{2}\right) \leqslant \Psi(\xi \sqcap+(1-\xi) \nu) \tilde{+} \Psi((1-\xi) \sqcap+\xi v)$.

Therefore, for every $\beta \in[0,1]$, we have

$$
\begin{gathered}
\frac{1}{h\left(\frac{1}{2}\right)} \Psi *\left(\frac{\sqcap+v}{2}, \beta\right) \\
\leq \Psi *(\xi \sqcap+(1-\xi) \nu, \beta)+\Psi *((1-\xi) \sqcap+\xi \nu, \beta), \\
\frac{1}{h\left(\frac{1}{2}\right)} \Psi^{*}\left(\frac{\sqcap+v}{2}, \beta\right) \\
\leq \Psi^{*}(\xi \sqcap+(1-\xi) \nu, \beta)+\Psi^{*}((1-\xi) \sqcap+\xi \nu, \beta) .
\end{gathered}
$$

Then 


$$
\begin{gathered}
\frac{1}{h\left(\frac{1}{2}\right)} \int_{0}^{1} \Psi_{*}\left(\frac{\mu+\nu}{2}, \beta\right) \mathrm{d} \xi \leq \int_{0}^{1} \Psi_{*}(\xi \mu+(1-\xi) \nu, \beta) \mathrm{d} \xi \\
\quad+\int_{0}^{1} \Psi_{*}((1-\xi) \nu+\xi \nu, \beta) \mathrm{d} \xi, \\
\frac{1}{h\left(\frac{1}{2}\right)} \int_{0}^{1} \Psi^{*}\left(\frac{\mu+\nu}{2}, \beta\right) \mathrm{d} \xi \leq \int_{0}^{1} \Psi^{*}(\xi \mu+(1-\xi) \nu, \beta) \mathrm{d} \xi \\
+\int_{0}^{1} \Psi^{*}((1-\xi) \mu+\xi \nu, \beta) \mathrm{d} \xi .
\end{gathered}
$$

It follows that

$$
\begin{aligned}
& \frac{1}{h\left(\frac{1}{2}\right)} \Psi_{*}\left(\frac{\mu+v}{2}, \beta\right) \leq \frac{2}{v-\mu} \int_{\mu}^{v} \Psi_{*}(z, \beta) \mathrm{d} z, \\
& \frac{1}{h\left(\frac{1}{2}\right)} \Psi^{*}\left(\frac{\mu+v}{2}, \beta\right) \leq \frac{2}{v-\mu} \int_{\mu}^{v} \Psi^{*}(z, \beta) \mathrm{d} z .
\end{aligned}
$$

That is

$$
\begin{aligned}
& \frac{1}{h\left(\frac{1}{2}\right)}\left[\Psi_{*}\left(\frac{\mu+v}{2}, \beta\right), \Psi^{*}\left(\frac{\mu+v}{2}, \beta\right)\right] \\
& \leq_{I} \frac{2}{v-\mu}\left[\int_{\mu}^{v} \Psi_{*}(\mu, \beta) \mathrm{d} z, \int_{\mu}^{v} \Psi^{*}(z, \beta) \mathrm{d} z\right] .
\end{aligned}
$$

Thus,

$$
\frac{1}{2 h\left(\frac{1}{2}\right)} \Psi\left(\frac{\mu+v}{2}\right) \leqslant \frac{1}{v-\mu}(\mathrm{FR}) \int_{\mu}^{v} \Psi(z) \mathrm{d} z .
$$

In a similar way as above, we have

$$
\frac{1}{v-\mu}(\mathrm{FR}) \int_{\mu}^{v} \Psi(z) \mathrm{d} z \leqslant[\Psi(\mu) \tilde{+} \Psi(\nu)] \int_{0}^{1} h(\xi) \mathrm{d} \xi .
$$

Combining (16) and (17), we have

$$
\begin{aligned}
& \frac{1}{2 h\left(\frac{1}{2}\right)} \Psi\left(\frac{\mu+v}{2}\right) \leqslant \frac{1}{v-\mu}(\mathrm{FR}) \int_{\mu}^{v} \Psi(z) \mathrm{d} z \\
& \leqslant[\Psi(\mu) \tilde{+} \Psi(\nu)] \int_{0}^{1} h(\xi) \mathrm{d} \xi .
\end{aligned}
$$

Hence, the required result.

Remark 3.1. If $h(\xi)=\xi^{s}$, then Theorem 3.1 reduces to the result for $s$-convex FIVF which is also new one: $2^{s-1} \Psi\left(\frac{\mu+v}{2}\right) \leqslant \frac{1}{v-\mu}(\mathrm{FR}) \int_{\mu}^{v} \Psi(z) \mathrm{d} z$

$\preccurlyeq \frac{1}{s+1}[\Psi(\mu) \tilde{+} \Psi(v)]$.

If $h(\xi)=\xi$, then Theorem 3.1 reduces to the result for convex FIVF which is also new one:

$\Psi\left(\frac{\mu+v}{2}\right) \leqslant \frac{1}{v-\mu}(\mathrm{FR}) \int_{\mu}^{v} \Psi(z) \mathrm{d} z \leqslant \frac{\Psi(\mu) \tilde{+} \Psi(\nu)}{2}$.

If $h(\xi) \equiv 1$ then Theorem 3.1 reduces to the result for $P$ FIVF which is also new one:

$\frac{1}{2} \Psi\left(\frac{\mu+v}{2}\right) \preccurlyeq \frac{1}{\nu-\mu}(F R) \int_{\mu}^{v} \Psi(z) d z \preccurlyeq \Psi(\mu) \tilde{+} \Psi(v)$.

If $\Psi_{*}(z, \beta)=\Psi^{*}(z, \beta)$ with $\beta=1$, then Theorem 3.1 reduces to the result for classical $h$-convex function, see [29]:

$\frac{1}{2 h\left(\frac{1}{2}\right)} \Psi\left(\frac{\mu+v}{2}\right) \leq \frac{1}{v-\mu}(R) \int_{\mu}^{v} \Psi(z) \mathrm{d} z$

$\leq[\Psi(\mu)+\Psi(v)] \int_{0}^{1} h(\xi) \mathrm{d} \xi$.

If $\Psi_{*}(z, \beta)=\Psi^{*}(z, \beta)$ with $\beta=1$ and $h(\xi)=\xi^{s}$, then Theorem 3.1 reduces to the result for classical $s$-convex function, see [21]:

$2^{s-1} \Psi\left(\frac{\mu+v}{2}\right) \leq \frac{1}{v-\mu}(R) \int_{\mu}^{\nu} \Psi(z) \mathrm{d} z$

$\leq \frac{1}{s+1}[\Psi(\mu)+\Psi(v)]$

If $\Psi_{*}(z, \beta)=\Psi^{*}(z, \beta)$ with $\beta=1$ and $h(\xi)=\xi$, then Theorem 3.1 reduces to the result for classical convex function, see $[16,17]$ :

$\Psi\left(\frac{\mu+v}{2}\right) \leq \frac{1}{v-\mu}(R) \int_{\mu}^{v} \Psi(\mu) \mathrm{d} z \leq \frac{\Psi(\mu)+\Psi(v)}{2}$.

If $\Psi_{*}(z, \beta)=\Psi^{*}(z, \beta)$ with $\beta=1$ and $h(\xi) \equiv 1$ then Theorem 3.1 reduces to the result for classical $P$-convex function, see [14]: 
$\frac{1}{2} \Psi\left(\frac{\mu+v}{2}\right) \leq \frac{1}{v-\mu}(R) \int_{\mu}^{v} \Psi(z) \mathrm{d} z \leq \Psi(\mu)+\Psi(v)$.

Example 3.1 We consider $h(\xi)=\xi$, for $\xi \in[0,1]$, and the FIVF $\Psi:[\mu, v]=[0,2] \rightarrow \mathbb{F}_{C}(\mathbb{R})$ defined by,

$\Psi(z)(\sigma)=\left\{\begin{array}{c}\frac{\sigma}{2 z^{2}} \sigma \in\left[0,2 z^{2}\right] \\ \frac{4 z^{2}-\sigma}{2 z^{2}} \sigma \in\left(2 z^{2}, 4 z^{2}\right] \\ \text { 0otherwise, }\end{array}\right.$

Then, for each $\beta \in[0,1]$, we have $\Psi_{\beta}(z)=\left[2 \beta z^{2},(4-2 \beta) z^{2}\right]$. Since end point functions $\Psi_{*}(z, \beta)=2 \beta z^{2}, \Psi^{*}(z, \beta)=(4-2 \beta) z^{2}$ are $h$-convex functions for each $\beta \in[0,1]$. Hence $\Psi(z)$ is $h$-convex FIVF. We now computing the following

$\frac{1}{2 h\left(\frac{1}{2}\right)} \Psi_{*}\left(\frac{\mu+v}{2}, \beta\right) \leq \frac{1}{v-\mu} \int_{\mu}^{v} \Psi_{*}(z, \beta) \mathrm{d} z$

$\leq\left[\Psi_{*}(\mu, \beta)+\Psi_{*}(\nu, \beta)\right] \int_{0}^{1} h(\xi) \mathrm{d} \xi$.

$\frac{1}{2 h\left(\frac{1}{2}\right)} \Psi_{*}\left(\frac{\mu+v}{2}, \beta\right)=\Psi_{*}(1, \beta)=2 \beta$,

$\frac{1}{v-\mu} \int_{\mu}^{v} \Psi_{*}(z, \beta) \mathrm{d} z=\frac{1}{2} \int_{0}^{2} 2 \beta z^{2} \mathrm{~d} z=\frac{8 \beta}{3}$,

$\left[\Psi_{*}(\mu, \beta)+\Psi_{*}(\nu, \beta)\right] \int_{0}^{1} h(\xi) \mathrm{d} \xi=4 \beta$,

for all $\beta \in[0,1]$. That means

$2 \beta \leq \frac{8 \beta}{3} \leq 4 \beta$.

Similarly, it can be easily show that

$$
\begin{aligned}
& \frac{1}{2 h\left(\frac{1}{2}\right)} \Psi^{*}\left(\frac{\mu+v}{2}, \beta\right) \leq \frac{1}{v-\mu} \int_{\mu}^{v} \Psi^{*}(z, \beta) \mathrm{d} z \\
& \leq\left[\Psi^{*}(\nu, \beta)+\Psi^{*}(\nu, \beta)\right] \int_{0}^{1} h(\xi) \mathrm{d} \xi .
\end{aligned}
$$

for all $\beta \in[0,1]$, such that

$$
\frac{1}{2 h\left(\frac{1}{2}\right)} \Psi^{*}\left(\frac{\mu+v}{2}, \beta\right)=\Psi_{*}(1, \beta)=(4-2 \beta),
$$

$$
\frac{1}{v-\mu} \int_{\mu}^{v} \Psi^{*}(z, \beta) \mathrm{d} z=\frac{1}{2} \int_{0}^{2}(4-2 \beta) z^{2} \mathrm{~d} z=\frac{4(4-2 \beta)}{3}
$$

$\left[\Psi^{*}(\mu, \beta)+\Psi^{*}(\nu, \beta)\right] \int_{0}^{1} h(\xi) \mathrm{d} \xi=2(4-2 \beta)$.

From which, we have

$(4-2 \beta) \leq \frac{4(4-2 \beta)}{3} \leq 2(4-2 \beta)$

that is

$[2 \beta,(4-2 \beta)] \leq_{I}\left[\frac{8 \beta}{3}, \frac{4(4-2 \beta)}{3}\right] \leq_{I}[4 \beta, 2(4-2 \beta)]$,

for all $\beta \in[0,1]$.

Hence,

$\frac{1}{2 h\left(\frac{1}{2}\right)} \Psi\left(\frac{\mu+v}{2}\right) \leqslant \frac{1}{v-\mu}(\mathrm{FR}) \int_{\mu}^{v} \Psi(z) \mathrm{d} z$

$\preccurlyeq[\Psi(\mu) \tilde{+} \Psi(v)] \int_{0}^{1} h(\xi) \mathrm{d} \xi$

Theorem 3.2. Let $\Psi:[\mu, v] \rightarrow \mathbb{F}_{C}(\mathbb{R})$ be a $h$-convex FIVF with non-negative real valued function $h:[0,1] \rightarrow \mathbb{R}$ and $h\left(\frac{1}{2}\right) \neq 0$, and for all $\beta \in[0,1], \Psi_{\beta}: K \subset \mathbb{R} \rightarrow \mathcal{K}_{C}^{+} \subset \mathcal{K}_{C}$ represent the family of IVFs through $\beta$-levels. If $\Psi \in \mathcal{F} \mathcal{R}_{([\mu, \nu], \beta)}$, then

$\frac{1}{4\left[h\left(\frac{1}{2}\right)\right]^{2}} \Psi\left(\frac{\mu+v}{2}\right) \leqslant \triangleright_{2} \preccurlyeq \frac{1}{v-\mu}(\mathrm{FR}) \int_{\mu}^{\nu} \Psi(z) \mathrm{d} z \leqslant \triangleright_{1}$

$\preccurlyeq[\Psi(\mu) \tilde{+} \Psi(v)]\left[\frac{1}{2}+h\left(\frac{1}{2}\right)\right] \int_{0}^{1} h(\xi) \mathrm{d} \xi$,

where 
$\triangleright_{1}=\left[\frac{\Psi(\mu) \tilde{+} \Psi(v)}{2} \tilde{+} \Psi\left(\frac{\mu+v}{2}\right)\right] \int_{0}^{1} h(\xi) \mathrm{d} \xi$,

$\triangleright_{2}=\frac{1}{4 h\left(\frac{1}{2}\right)}\left[\Psi\left(\frac{3 \mu+v}{4}\right) \tilde{+} \Psi\left(\frac{\mu+3 v}{4}\right)\right]$,

and $\triangleright_{1}=\left[\triangleright_{1 *}, \triangleright_{1}^{*}\right], \triangleright_{2}=\left[\triangleright_{2 *}, \triangleright_{2}^{*}\right]$.

Proof. Take $\left[\mu, \frac{\mu+v}{2}\right]$, we have

$\frac{1}{h\left(\frac{1}{2}\right)} \Psi\left(\frac{\xi \mu+(1-\xi) \frac{\mu+\nu}{2}}{2}+\frac{(1-\xi) \mu+\xi \frac{\mu+v}{2}}{2}\right)$

$\preccurlyeq \Psi\left(\xi \mu+(1-\xi) \frac{\mu+v}{2}\right) \tilde{+} \Psi\left((1-\xi) \mu+\xi \frac{\mu+v}{2}\right)$.

Therefore, for every $\beta \in[0,1]$, we have

$\frac{1}{h\left(\frac{1}{2}\right)} \Psi_{*}\left(\frac{\xi \mu+(1-\xi) \frac{\mu+v}{2}}{2}+\frac{(1-\xi) \mu+\xi \frac{\mu+v}{2}}{2}, \beta\right)$

$\leq \Psi_{*}\left(\xi \mu+(1-\xi) \frac{\mu+v}{2}, \beta\right)+\Psi_{*}\left((1-\xi) \mu+\xi \frac{\mu+v}{2}, \beta\right)$,

$$
\frac{1}{h\left(\frac{1}{2}\right)} \Psi *\left(\frac{\xi \mu+(1-\xi) \frac{\mu+\nu}{2}}{2}+\frac{(1-\xi) \mu+\xi \frac{\mu+\nu}{2}}{2}, \beta\right)
$$

$\leq \Psi^{*}\left(\xi \mu+(1-\xi) \frac{\mu+\nu}{2}, \beta\right)+\Psi^{*}\left((1-\xi) \mu+\xi \frac{\mu+\nu}{2}, \beta\right)$.

In consequence, we obtain

$\frac{1}{4 h\left(\frac{1}{2}\right)} \Psi_{*}\left(\frac{3 \mu+v}{4}, \beta\right) \leq \frac{1}{v-\mu} \int_{\mu}^{\frac{\mu+v}{2}} \Psi_{*}(z, \beta) \mathrm{d} z$,

$\frac{1}{4 h\left(\frac{1}{2}\right)} \Psi^{*}\left(\frac{3 \mu+v}{4}, \beta\right) \leq \frac{1}{v-\mu} \int_{\mu}^{\frac{\mu+v}{2}} \Psi^{*}(z, \beta) \mathrm{d} z$.

That is

$\frac{1}{4 h\left(\frac{1}{2}\right)}\left[\Psi_{*}\left(\frac{3 \mu+v}{4}, \beta\right), \Psi^{*}\left(\frac{3 \mu+v}{4}, \beta\right)\right]$

$\leq_{I} \frac{1}{v-\mu}\left[\int_{\mu}^{\frac{\mu+v}{2}} \Psi_{*}(z, \beta) \mathrm{d} z, \int_{\mu}^{\frac{\mu+v}{2}} \Psi^{*}(z, \beta) \mathrm{d} z\right]$.

It follows that $\frac{1}{4 h\left(\frac{1}{2}\right)} \Psi\left(\frac{3 \mu+v}{4}\right) \leqslant \frac{1}{v-\mu} \int_{\mu}^{\frac{\mu+v}{2}} \Psi(z) \mathrm{d} z$.

In a similar way as above, we have

$\frac{1}{4 h\left(\frac{1}{2}\right)} \Psi\left(\frac{\mu+3 v}{4}\right) \leqslant \frac{1}{v-\mu} \int_{\frac{\mu+v}{2}}^{v} \Psi(z) \mathrm{d} z$.

Combining (18) and (19), we have

$\frac{1}{4 h\left(\frac{1}{2}\right)}\left[\Psi\left(\frac{3 \mu+v}{4}\right) \tilde{+} \Psi\left(\frac{\mu+3 v}{4}\right)\right] \preccurlyeq \frac{1}{v-\mu} \int_{\mu}^{v} \Psi(z) \mathrm{d} z$.

Using Theorem 3.1, we have

$\frac{1}{4\left[h\left(\frac{1}{2}\right)\right]^{2}} \Psi\left(\frac{\mu+v}{2}\right)=\frac{1}{4\left[h\left(\frac{1}{2}\right)\right]^{2}} \Psi\left(\frac{1}{2} \cdot \frac{3 \mu+v}{4}+\frac{1}{2} \cdot \frac{\mu+3 v}{4}\right)$.

Therefore, for every $\beta \in[0,1]$, we have

$\frac{1}{4\left[h\left(\frac{1}{2}\right)\right]^{2}} \Psi_{*}\left(\frac{\mu+v}{2}, \beta\right)=\frac{1}{4\left[h\left(\frac{1}{2}\right)\right]^{2}} \Psi_{*}\left(\frac{1}{2} \cdot \frac{3 \mu+v}{4}+\frac{1}{2} \cdot \frac{\mu+3 v}{4}, \beta\right)$,
$\frac{1}{4\left[h\left(\frac{1}{2}\right)\right]^{2}} \Psi^{*}\left(\frac{\mu+v}{2}, \beta\right)=\frac{1}{4\left[h\left(\frac{1}{2}\right)\right]^{2}} \Psi^{*}\left(\frac{1}{2} \cdot \frac{3 \mu+v}{4}+\frac{1}{2} \cdot \frac{\mu+3 v}{4}, \beta\right)$,

$\leq \frac{1}{4\left[h\left(\frac{1}{2}\right)\right]^{2}}\left[h\left(\frac{1}{2}\right) \Psi_{*}\left(\frac{3 \mu+v}{4}, \beta\right)+h\left(\frac{1}{2}\right) \Psi_{*}\left(\frac{\mu+3 v}{4}, \beta\right)\right]$,

$\leq \frac{1}{4\left[h\left(\frac{1}{2}\right)\right]^{2}}\left[h\left(\frac{1}{2}\right) \Psi^{*}\left(\frac{3 \mu+v}{4}, \beta\right)+h\left(\frac{1}{2}\right) \Psi^{*}\left(\frac{\mu+3 v}{4}, \beta\right)\right]$,

$=\triangleright_{2 *}$,

$=\triangleright_{2}^{*}$,

$\leq \frac{1}{v-\mu} \int_{\mu}^{v} \Psi_{*}(z, \beta) \mathrm{d} z$

$\leq \frac{1}{v-\mu} \int_{\mu} \Psi^{*}(z, \beta) \mathrm{d} z$

$\leq\left[\frac{\Psi_{*}(\mu, \beta)+\Psi_{*}(\nu, \beta)}{2}+\Psi_{*}\left(\frac{\mu+\nu}{2}, \beta\right)\right] \int_{0}^{1} h(\xi) \mathrm{d} \xi$,

$\leq\left[\frac{\Psi^{*}(\mu, \beta)+\Psi^{*}(v, \beta)}{2}+\Psi^{*}\left(\frac{\mu+v}{2}, \beta\right)\right] \int_{0}^{1} h(\xi) \mathrm{d} \xi$,

$=\triangleright_{1 *}$,

$=\triangleright_{1}^{*}$, 
$\leq\left[\frac{\Psi_{*}(\mu, \beta)+\Psi_{*}(\nu, \beta)}{2}+h(\xi)\left(\begin{array}{c}\Psi_{*}(\mu, \beta)+ \\ \Psi_{*}(\nu, \beta)\end{array}\right)\right] \int_{0}^{1} h(\xi) \mathrm{d} \xi$,

$\leq\left[\frac{\Psi^{*}(\mu, \beta)+\Psi^{*}(\nu, \beta)}{2}+h(\xi)\left(\begin{array}{c}\Psi^{*}(\mu, \beta)+ \\ \Psi^{*}(\nu, \beta)\end{array}\right)\right]_{0}^{1} h(\xi) \mathrm{d} \xi$,

$=\left[\Psi_{*}(\mu, \beta)+\Psi_{*}(\nu, \beta)\right]\left[\frac{1}{2}+h\left(\frac{1}{2}\right)\right] \int_{0}^{1} h(\xi) \mathrm{d} \xi$,

$=\left[\Psi^{*}(\mu, \beta)+\Psi^{*}(\nu, \beta)\right]\left[\frac{1}{2}+h\left(\frac{1}{2}\right)\right] \int_{0}^{1} h(\xi) \mathrm{d} \xi$,

that is

$\frac{1}{4\left[h\left(\frac{1}{2}\right)\right]^{2}} \Psi\left(\frac{\mu+v}{2}\right) \leqslant \triangleright_{2} \preccurlyeq \frac{1}{v-\mu}(\mathrm{FR}) \int_{\mu}^{v} \Psi(z) \mathrm{d} z \preccurlyeq \triangleright_{1}$

$\preccurlyeq[\Psi(\mu) \tilde{+} \Psi(\nu)]\left[\frac{1}{2}+h\left(\frac{1}{2}\right)\right] \int_{0}^{1} h(\xi) \mathrm{d} \xi$.

Hence, the proof has been completed.

Example 3.2. We consider $h(\xi)=\xi$, for $\xi \in[0,1]$, and the FIVF $\Psi:[\mu, v]=[0,2] \rightarrow \mathbb{F}_{C}(\mathbb{R})$ defined by, $\Psi_{\beta}(z)=\left[2 \beta z^{2},(4-2 \beta) z^{2}\right]$, as in Example 3.1, then $\Psi(z)$ is $h$-convex FIVF and satisfying (10). We have $\Psi_{*}(z, \beta)=\beta z$ and $\Psi^{*}(z, \beta)=(4-2 \beta) z$. We now computing the following.

$$
\begin{gathered}
{\left[\Psi_{*}(\mu, \beta)+\Psi_{*}(\nu, \beta)\right]\left[\frac{1}{2}+h\left(\frac{1}{2}\right)\right] \int_{0}^{1} h(\xi) \mathrm{d} \xi=4 \beta,} \\
{\left[\Psi^{*}(\mu, \beta)+\Psi^{*}(\nu, \beta)\right]\left[\frac{1}{2}+h\left(\frac{1}{2}\right)\right] \int_{0}^{1} h(\xi) \mathrm{d} \xi=4(2-\beta),} \\
\triangleright_{1 *}=\left[\frac{\Psi_{*}(\mu, \beta)+\Psi_{*}(v, \beta)}{2}+\Psi_{*}\left(\frac{\mu+v}{2}, \beta\right)\right] \int_{0}^{1} h(\xi) \mathrm{d} \xi \\
=3 \beta, \\
\triangleright_{1}^{*}=\left[\frac{\Psi^{*}(\mu, \beta)+\Psi^{*}(\nu, \beta)}{2}+\Psi^{*}\left(\frac{\mu+v}{2}, \beta\right)\right] \int_{0}^{1} h(\xi) \mathrm{d} \xi \\
\triangleright_{2 *}=\frac{1}{4\left[h\left(\frac{1}{2}\right)\right]^{2}}\left[h\left(\frac{1}{2}\right) \Psi_{*}\left(\frac{3 \mu+v}{4}, \beta\right)+h\left(\frac{1}{2}\right) \Psi_{*}\left(\frac{\mu+3 v}{4}, \beta\right)\right] \\
\quad=\frac{5}{2} \beta, \\
\triangleright_{2}^{*}=\frac{1}{4\left[h\left(\frac{1}{2}\right)\right]^{2}}\left[h\left(\frac{1}{2}\right) \Psi^{*}\left(\frac{3 \mu+v}{4}, \beta\right)+h\left(\frac{1}{2}\right) \Psi^{*}\left(\frac{\mu+3 v}{4}, \beta\right)\right] \\
=\frac{5}{2}(2-\beta) .
\end{gathered}
$$

Then we obtain that

$$
\begin{gathered}
2 \beta \leq \frac{5}{2} \beta \leq \frac{8 \beta}{3} \leq 3 \beta \leq 4 \beta \\
2(2-\beta) \leq \frac{5}{2}(2-\beta) \leq \frac{8(2-\beta)}{3} \leq 3(2-\beta) \leq 4(2-\beta) .
\end{gathered}
$$

Hence, Theorem 3.2 is verified.

Following results find the new versions of $\mathrm{H}-\mathrm{H}$ inequalities for the product of two $h$-convex FIVFs.

Theorem 3.3. Let $\Psi, \mathcal{J}:[\mu, v] \rightarrow \mathbb{F}_{C}(\mathbb{R})$ be two $h$ -convex FIVFs with non-negative real valued functions $h_{1}, h_{2}:[0,1] \rightarrow \mathbb{R}$, and for all $\beta \in[0,1]$, $\Psi_{\beta}: K \subset \mathbb{R} \rightarrow \mathcal{K}_{C}^{+} \subset \mathcal{K}_{C}$ represent the family of IVFs through $\beta$-levels. If $\Psi, \mathcal{J}$ and $\Psi \mathcal{J} \in \mathcal{F} \mathcal{R}_{([\mu, \nu], \beta)}$, then

$$
\frac{1}{v-\mu}(\mathrm{FR}) \int_{\mu}^{v} \Psi(\mu) \tilde{\times} \mathcal{J}(z) \mathrm{d} z
$$

$\preccurlyeq \mathcal{M}(\mu, v) \int_{0}^{1} h_{1}(\xi) h_{2}(\xi) \mathrm{d} \xi$

$\tilde{+} \mathcal{N}(\mu, v) \int_{0}^{1} h_{1}(\xi) h_{2}(1-\xi) \mathrm{d} \xi$,

where $\mathcal{M}(\mu, v)=\Psi(\mu) \tilde{\times} \mathcal{J}(\mu) \tilde{+} \Psi(v) \tilde{\times} \mathcal{J}(v)$, $\mathcal{N}(\mu, v)=\Psi(\mu) \tilde{\times} \mathcal{J}(v) \tilde{+} \Psi(v) \tilde{\times} \mathcal{J}(\mu), \quad$ a $n \quad d$ $\mathcal{M}_{\beta}(\mu, v)=\left[\mathcal{M} *((\mu, v), \beta), \mathcal{M}^{*}((\mu, v), \beta)\right] \quad a n d$ $\mathcal{N}_{\beta}(\mu, v)=\left[\mathcal{N} *((\mu, v), \beta), \mathcal{N}^{*}((\mu, v), \beta)\right]$.

Example 3.3. We consider $h_{1}(\xi)=\xi, h_{2}(\xi) \equiv 1$, for $\xi \in[0,1]$, and the fuzzy interval-valued functions $\Psi, \mathcal{J}:[\mu, \nu]=[0,1] \rightarrow \mathbb{F}_{C}(\mathbb{R})$ defined by,

$$
\begin{aligned}
& \Psi(z)(\sigma)=\left\{\begin{array}{c}
\frac{\sigma}{2 z^{2}} \sigma \in\left[0,2 z^{2}\right] \\
\frac{4 z^{2}-\sigma}{2 z^{2}} \sigma \in\left(2 z^{2}, 4 z^{2}\right] \\
\text { Ootherwise, }
\end{array}\right. \\
& \mathcal{J}(z)(\sigma)=\left\{\begin{array}{c}
\frac{\sigma}{z} \sigma \in[0, z] \\
\frac{2 z-\sigma}{z} \sigma \in(z, 2 z] \\
\text { Ootherwise, }
\end{array}\right.
\end{aligned}
$$

Then, for each $\beta \in[0,1]$, we have $\Psi_{\beta}(z)=\left[2 \beta z^{2},(4-2 \beta) z^{2}\right]$ and $\mathcal{J}_{\beta}(z)=[\beta z,(2-\beta) z]$. Since end point functions $\Psi_{*}(z, \beta)=2 \beta z^{2}, \Psi^{*}(z, \beta)=(4-2 \beta) z^{2}$ and $\mathcal{J} *(z, \beta)=\beta z, \mathcal{J}^{*}(z, \beta)=(2-\beta) z h_{1}$ and $h_{2}$-convex functions for each $\beta \in[0,1]$, respectively. Hence $\Psi, \mathcal{J}$ both are $h$-convex FIVFs. We now computing the following 
$\frac{1}{v-\mu} \int_{\nu}^{v} \Psi_{*}(z, \beta) \times \mathcal{J} *(z, \beta) \mathrm{d} z=\frac{\beta^{2}}{2}$,

$\frac{1}{v-\mu} \int_{\mu} \Psi^{*}(z, \beta) \times \mathcal{J}^{*}(z, \beta) \mathrm{d} z=\frac{(2-\beta)^{2}}{2}$,

$\mathcal{M} *((\mu, \nu), \beta) \int_{0}^{1} h_{1}(\xi) h_{2}(\xi) \mathrm{d} \xi=\beta^{2}$,

$\mathcal{M}^{*}((\mu, \nu), \beta) \int_{0}^{1} h_{1}(\xi) h_{2}(\xi) \mathrm{d} \xi=(2-\beta)^{2}$,

$\mathcal{N} *((\mu, \nu), \beta) \int_{0}^{1} h_{1}(\xi) h_{2}(1-\xi) \mathrm{d} \xi=0$

$\mathcal{N}^{*}((\mu, \nu), \beta) \int_{0} h_{1}(\xi) h_{2}(1-\xi) \mathrm{d} \xi=0$

for each $\beta \in[0,1]$, that means

$$
\frac{\beta^{2}}{2} \leq \beta^{2}+0=\beta^{2},
$$

$\frac{(2-\beta)^{2}}{2} \leq(2-\beta)^{2}+0=(2-\beta)^{2}$,

Hence, Theorem 3.3 is demonstrated.

Theorem 3.4 Let $\Psi, \mathcal{J}:[\sqcap, v] \rightarrow \mathbb{F}_{C}(\mathbb{R})$ be two h-convex FIVFs with non-negative real valued functions $h_{1}, h_{2}:[0,1] \rightarrow \mathbb{R}$, respectively and $h_{1}\left(\frac{1}{2}\right) h_{2}\left(\frac{1}{2}\right) \neq 0$, and for all $\beta \in[0,1], \Psi_{\beta}: K \subset \mathbb{R} \rightarrow \mathcal{K}_{C}{ }^{+} \subset \mathcal{K}_{C}$ represent the family of IVFs through $\beta$-levels. If $\Psi \mathcal{J} \in \mathcal{F} \mathcal{R}_{([\sqcap, \nu], \beta)}$, then

$\frac{1}{2 h_{1}\left(\frac{1}{2}\right) h_{2}\left(\frac{1}{2}\right)} \Psi\left(\frac{\Pi+v}{2}\right) \tilde{\times} \mathcal{J}\left(\frac{\Pi+v}{2}\right)$

$\preccurlyeq \frac{1}{v-\sqcap}(F R) \int_{\sqcap}^{v} \Psi(\ddagger) \tilde{\times} \mathcal{J}(\ddagger) \mathrm{d} \ddagger$

$\tilde{+} \mathcal{M}(\sqcap, v) \int_{0}^{1} h_{1}(\xi) h_{2}(1-\xi) \mathrm{d} \xi \tilde{+} \mathcal{N}(\sqcap, v) \int_{0}^{1} h_{1}(\xi) h_{2}(\xi) \mathrm{d} \xi$,

where $\quad \mathcal{M}(\sqcap, v)=\Psi(\sqcap) \underset{\sim}{\sim} \mathcal{\sim} \mathcal{J}(\sqcap) \tilde{+} \Psi(v) \tilde{\times} \mathcal{J}(v)$, $\mathcal{N}(\sqcap, v)=\Psi(\sqcap) \tilde{\times} \mathcal{J}(v)+\Psi(v) \times \mathcal{\times} \mathcal{J}(\sqcap), \quad a n d$ $\mathcal{M}_{\beta}(\sqcap, v)=\left[\mathcal{M} *((\sqcap, v), \beta), \mathcal{M}^{*}((\sqcap, v), \beta)\right] \quad$ a $n d$ $\mathcal{N}_{\beta}(\sqcap, v)=\left[\mathcal{N} *((\sqcap, v), \beta), \mathcal{N}^{*}((\sqcap, v), \beta)\right]$.

Proof. By hypothesis, for each $\beta \in[0,1]$, we have

$$
\begin{gathered}
\Psi *\left(\frac{\sqcap+v}{2}, \beta\right) \times \mathcal{J} *\left(\frac{\sqcap+v}{2}, \beta\right) \\
\Psi^{*}\left(\frac{\sqcap+v}{2}, \beta\right) \times \mathcal{J}^{*}\left(\frac{\sqcap+v}{2}, \beta\right)
\end{gathered}
$$

$\leq h_{1}\left(\frac{1}{2}\right) h_{2}\left(\frac{1}{2}\right)\left[\begin{array}{c}\Psi *\left(\begin{array}{c}\xi u+ \\ (1-\xi) \nu, \beta\end{array}\right) \times \mathcal{J} *\left(\begin{array}{c}\xi u+ \\ (1-\xi) \nu, \beta\end{array}\right) \\ +\Psi *\left(\begin{array}{c}\xi u+ \\ (1-\xi) \nu, \beta\end{array}\right) \times \mathcal{J} *\left(\begin{array}{c}(1-\xi) u+ \\ \xi \nu, \beta\end{array}\right)\end{array}\right]$

$+h_{1}\left(\frac{1}{2}\right) h_{2}\left(\frac{1}{2}\right)\left[\begin{array}{c}\Psi *\left(\begin{array}{c}(1-\xi) u+ \\ \xi \nu, \beta\end{array}\right) \times \mathcal{J} *\left(\begin{array}{c}\xi u+ \\ (1-\xi) \nu, \beta\end{array}\right) \\ +\Psi *\left(\begin{array}{c}(1-\xi) u+ \\ \xi \nu, \beta\end{array}\right) \times \mathcal{J} *\left(\begin{array}{c}(1-\xi) u+ \\ \xi \nu, \beta\end{array}\right)\end{array}\right]$,

$\leq h_{1}\left(\frac{1}{2}\right) h_{2}\left(\frac{1}{2}\right)\left[\begin{array}{c}\Psi^{*}\left(\begin{array}{c}\xi u+ \\ (1-\xi) \nu, \beta\end{array}\right) \times \mathcal{J}^{*}\left(\begin{array}{c}\xi u+ \\ (1-\xi) \nu, \beta\end{array}\right) \\ +\Psi^{*}\left(\begin{array}{c}\xi u+ \\ (1-\xi) \nu, \beta\end{array}\right) \times \mathcal{J}^{*}\left(\begin{array}{c}(1-\xi) u+ \\ \xi \nu, \beta\end{array}\right)\end{array}\right]$

$+h_{1}\left(\frac{1}{2}\right) h_{2}\left(\frac{1}{2}\right)\left[\begin{array}{c}\Psi^{*}\left(\begin{array}{c}(1-\xi) u+ \\ \xi \nu, \beta\end{array}\right) \times \mathcal{J}^{*}\left(\begin{array}{c}\xi u+ \\ (1-\xi) \nu, \beta\end{array}\right) \\ +\Psi^{*}\left(\begin{array}{c}(1-\xi) u+ \\ \xi \nu, \beta\end{array}\right) \times \mathcal{J}^{*}\left(\begin{array}{c}(1-\xi) u+ \\ \xi \nu, \beta\end{array}\right)\end{array}\right]$,

$\leq h_{1}\left(\frac{1}{2}\right) h_{2}\left(\frac{1}{2}\right)\left[\begin{array}{c}\Psi *\left(\begin{array}{c}\xi u+ \\ (1-\xi) \nu, \beta\end{array}\right) \times \mathcal{J} *\left(\begin{array}{c}\xi u+ \\ (1-\xi) \nu, \beta\end{array}\right) \\ +\Psi *\left(\begin{array}{c}(1-\xi) u+ \\ \xi \nu, \beta\end{array}\right) \times \mathcal{J} *\left(\begin{array}{c}(1-\xi) u+ \\ \xi \nu, \beta\end{array}\right)\end{array}\right]$

$$
+h_{1}\left(\frac{1}{2}\right) h_{2}\left(\frac{1}{2}\right)\left[\begin{array}{c}
\left(\begin{array}{c}
h_{1}(\xi) \Psi *(\sqcap, \beta)+ \\
h_{1}(1-\xi) \Psi *(\nu, \beta) \\
h_{2}(1-\xi) \mathcal{J} *(\sqcap, \beta)+ \\
h_{2}(\xi) \mathcal{J} *(\nu, \beta)
\end{array}\right) \\
\times\left(\begin{array}{c}
h_{1}(1-\xi) \Psi *(\sqcap, \beta)+ \\
h_{1}(\xi) \Psi *(\nu, \beta)
\end{array}\right) \\
\times\left(\begin{array}{c}
h_{2}(\xi) \mathcal{J} *(\sqcap, \beta)+ \\
h_{2}(1-\xi) \mathcal{J} *(\nu, \beta)
\end{array}\right)
\end{array}\right] \text {, }
$$

$\leq h_{1}\left(\frac{1}{2}\right) h_{2}\left(\frac{1}{2}\right)\left[\begin{array}{c}\Psi^{*}\left(\begin{array}{c}\xi u+ \\ (1-\xi) \nu, \beta\end{array}\right) \times \mathcal{J}^{*}\left(\begin{array}{c}\xi u+ \\ (1-\xi) \nu, \beta\end{array}\right) \\ +\Psi^{*}\left(\begin{array}{c}(1-\xi) u+ \\ \xi \nu, \beta\end{array}\right) \times \mathcal{J}^{*}\left(\begin{array}{c}(1-\xi) u+ \\ \xi \nu, \beta\end{array}\right)\end{array}\right]$

$$
+h_{1}\left(\frac{1}{2}\right) h_{2}\left(\frac{1}{2}\right)\left[\begin{array}{c}
\left(\begin{array}{c}
h_{1}(\xi) \Psi^{*}(\sqcap, \beta)+ \\
h_{1}(1-\xi) \Psi^{*}(\nu, \beta)
\end{array}\right) \\
\times\left(\begin{array}{c}
h_{2}(1-\xi) \mathcal{J}^{*}(\sqcap, \beta)+ \\
h_{2}(\xi) \mathcal{J}^{*}(\nu, \beta)
\end{array}\right) \\
+\left(\begin{array}{c}
h_{1}(1-\xi) \Psi^{*}(\sqcap, \beta)+ \\
h_{1}(\xi) \Psi^{*}(\nu, \beta)
\end{array}\right) \\
\times\left(\begin{array}{c}
h_{2}(\xi) \mathcal{J}^{*}(\sqcap, \beta)+ \\
h_{2}(1-\xi) \mathcal{J}^{*}(\nu, \beta)
\end{array}\right)
\end{array}\right],
$$

$=h_{1}\left(\frac{1}{2}\right) h_{2}\left(\frac{1}{2}\right)\left[\begin{array}{c}\Psi *\left(\begin{array}{c}\xi u+ \\ (1-\xi) \nu, \beta\end{array}\right) \times \mathcal{J} *\left(\begin{array}{c}\xi u+ \\ (1-\xi) \nu, \beta\end{array}\right) \\ +\Psi *\left(\begin{array}{c}(1-\xi) u+ \\ \xi \nu, \beta\end{array}\right) \times \mathcal{J} *\left(\begin{array}{c}(1-\xi) u+ \\ \xi \nu, \beta\end{array}\right)\end{array}\right]$ 


$$
\begin{aligned}
& +h_{1}\left(\frac{1}{2}\right) h_{2}\left(\frac{1}{2}\right)\left[\begin{array}{c}
\left\{\begin{array}{c}
h_{1}(\xi) h_{2}(\xi)+ \\
h_{1}(1-\xi) h_{2}(1-\xi)
\end{array}\right\} \mathcal{N} *((\sqcap, v), \beta) \\
+\left\{\begin{array}{c}
h_{1}(\xi) h_{2}(1-\xi)+ \\
h_{1}(1-\xi) h_{2}(\xi)
\end{array}\right\} \mathcal{M} *((\sqcap, \nu), \beta)
\end{array}\right], \\
& =h_{1}\left(\frac{1}{2}\right) h_{2}\left(\frac{1}{2}\right)\left[\begin{array}{c}
\Psi^{*}\left(\begin{array}{c}
\xi u+ \\
(1-\xi) \nu, \beta
\end{array}\right) \times \mathcal{J}^{*}\left(\begin{array}{c}
\xi u+ \\
(1-\xi) \nu, \beta
\end{array}\right) \\
+\Psi^{*}\left(\begin{array}{c}
(1-\xi) u+ \\
\xi \nu, \beta
\end{array}\right) \times \mathcal{J}^{*}\left(\begin{array}{c}
(1-\xi) u+ \\
\xi \nu, \beta
\end{array}\right)
\end{array}\right] \\
& +h_{1}\left(\frac{1}{2}\right) h_{2}\left(\frac{1}{2}\right)\left[\begin{array}{c}
\left\{\begin{array}{c}
h_{1}(\xi) h_{2}(\xi)+ \\
h_{1}(1-\xi) h_{2}(1-\xi)
\end{array}\right\} \mathcal{N}^{*}((\sqcap, \nu), \beta) \\
+\left\{\begin{array}{c}
h_{1}(\xi) h_{2}(1-\xi)+ \\
h_{1}(1-\xi) h_{2}(\xi)
\end{array}\right\} \mathcal{M}^{*}((\sqcap, \nu), \beta)
\end{array}\right],
\end{aligned}
$$

Integrating over $[0,1]$, we have

$$
\begin{gathered}
\frac{1}{2 h_{1}\left(\frac{1}{2}\right) h_{2}\left(\frac{1}{2}\right)} \Psi *\left(\frac{\Pi+v}{2}, \beta\right) \times \mathcal{J} *\left(\frac{\Pi+v}{2}, \beta\right) \\
\leq \frac{1}{v-\sqcap}(R) \int_{\Pi}^{v} \Psi *(\ddagger, \beta) \times \mathcal{J} *(\ddagger, \beta) \mathrm{d} \ddagger \\
+\mathcal{M} *((\sqcap, v), \beta) \int_{0}^{1} h_{1}(\xi) h_{2}(1-\xi) \mathrm{d} \xi \\
+\mathcal{N} *((\sqcap, \nu), \beta) \int_{0}^{1} h_{1}(\xi) h_{2}(\xi) \mathrm{d} \xi, \\
\frac{1}{2 h_{1}\left(\frac{1}{2}\right) h_{2}\left(\frac{1}{2}\right)} \Psi^{*}\left(\frac{\square+v}{2}, \beta\right) \times \mathcal{J}^{*}\left(\frac{\square+v}{2}, \beta\right) \\
\leq \frac{1}{v-\Pi}(R) \int_{\Pi}^{v} \Psi^{*}(\ddagger, \beta) \times \mathcal{J}^{*}(\ddagger, \beta) \mathrm{d} \ddagger \\
+\mathcal{M}^{*}((\sqcap, v), \beta) \int_{0}^{1} h_{1}(\xi) h_{2}(1-\xi) \mathrm{d} \xi \\
+\mathcal{N}^{*}((\sqcap, v), \beta) \int_{0}^{1} h_{1}(\xi) h_{2}(\xi) \mathrm{d} \xi,
\end{gathered}
$$

that is

$$
\begin{aligned}
& \frac{1}{2 h_{1}\left(\frac{1}{2}\right) h_{2}\left(\frac{1}{2}\right)} \Psi\left(\frac{\Pi+v}{2}\right) \tilde{\times} \mathcal{J}\left(\frac{\square+v}{2}\right) \\
& \preccurlyeq \frac{1}{v-\sqcap}(F R) \int_{\sqcap}^{v} \Psi(\ddagger) \tilde{\times} \mathcal{J}() \mathrm{d} \ddagger \\
& \tilde{+} \mathcal{M}(\sqcap, v) \int_{0}^{1} h_{1}(\xi) h_{2}(1-\xi) \mathrm{d} \xi \tilde{+} \mathcal{N}(\sqcap, \nu) \int_{0}^{1} h_{1}(\xi) h_{2}(\xi) \mathrm{d} \xi,
\end{aligned}
$$

Hence, the required result.

Example 3.4. We consider $h_{1}(\xi)=\xi, h_{2}(\xi) \equiv 1$, for $\xi \in[0,1]$, and the FIVFs $\Psi, \mathcal{J}:[\sqcap, \nu]=[0,1] \rightarrow \mathbb{F}_{C}(\mathbb{R})$, as in Example 3.3. Then, for each $\beta \in[0,1]$, we have $\Psi_{\beta}(\ddagger)=\left[2 \beta \ddagger^{2},(4-2 \beta) \ddagger^{2}\right]$ and $\mathcal{J}_{\beta}(\ddagger)=[\beta \ddagger,(2-\beta) \ddagger]$ and, $\Psi(\ddagger), \mathcal{J}(\ddagger)$ both are $h_{1}$-convex and $h_{2}$-convex FIVFs, respectively. We have $\Psi *(\ddagger, \beta)=2 \beta \ddagger^{2}, \Psi^{*}(\ddagger, \beta)=(4-2 \beta) \ddagger^{2}$ and $\mathcal{J} *(\ddagger, \beta)=\beta \ddagger, \mathcal{J}^{*}(\ddagger, \beta)=(2-\beta) \ddagger$. We now computing the following.

$$
\begin{aligned}
& \frac{1}{2 h_{1}\left(\frac{1}{2}\right) h_{2}\left(\frac{1}{2}\right)} \Psi *\left(\frac{\sqcap+v}{2}, \beta\right) \times \mathcal{J} *\left(\frac{\sqcap+v}{2}, \beta\right)=\frac{\beta^{2}}{4}, \\
& \frac{1}{2 h_{1}\left(\frac{1}{2}\right) h_{2}\left(\frac{1}{2}\right)} \Psi^{*}\left(\frac{\square+v}{2}, \beta\right) \times \mathcal{J}^{*}\left(\frac{\sqcap+v}{2}, \beta\right)=\frac{(2-\beta)^{2}}{4},
\end{aligned}
$$

$\frac{1}{v-\sqcap} \int_{\Pi}^{v} \Psi *(\ddagger, \beta) \times \mathcal{J} *(\ddagger, \beta) \mathrm{d} \ddagger=\frac{\beta^{2}}{2}$,

$\frac{1}{v-\sqcap} \int_{\Gamma}^{v} \Psi^{*}(\ddagger, \beta) \times \mathcal{J}^{*}(\ddagger, \beta) \mathrm{d} \ddagger=\frac{(2-\beta)^{2}}{2}$,

$\mathcal{M} *((\sqcap, \nu), \beta) \int_{0}^{1} h_{1}(\xi) h_{2}(1-\xi) \mathrm{d} \xi=\beta^{2}$, $\mathcal{M}^{*}((\sqcap, \nu), \beta) \int_{0}^{1} h_{1}(\xi) h_{2}(1-\xi) \mathrm{d} \xi=(2-\beta)^{2}$,

$\mathcal{N} *((\sqcap, \nu), \beta) \int_{0}^{1} h_{1}(\xi) h_{2}(\xi) \mathrm{d} \xi=0$, $\mathcal{N}^{*}((\sqcap, \nu), \beta) \int{ }_{0}^{1} h_{1}(\xi) h_{2}(\xi) \mathrm{d} \xi=0$,

for each $\beta \in[0,1]$, we conclude that

$$
\begin{gathered}
\frac{\beta^{2}}{4} \leq \frac{\beta^{2}}{2}+\beta^{2}+0=\frac{3 \beta}{2}, \\
\frac{(2-\beta)^{2}}{4} \leq \frac{(2-\beta)^{2}}{2}+(2-\beta)^{2}+0=\frac{3(2-\beta)^{2}}{2},
\end{gathered}
$$

hence, Theorem 3.4 is demonstrated.

We now give $H H$-Fej'er inequalities for $h$-convex FIVFs. Firstly, we obtain the second $H H$-Fej'er inequality for $h$-convex FIVF.

Theorem 3.5. Let $\Psi:[\sqcap, \nu] \rightarrow \mathbb{F}_{C}(\mathbb{R})$ be a h-convex FIVF with $\sqcap<v$ and $h:[0,1] \rightarrow \mathbb{R}^{+}$, and for all $\beta \in[0,1], \quad \Psi_{\beta}: K \subset \mathbb{R} \rightarrow \mathcal{K}_{C}{ }^{+} \subset \mathcal{K}_{C}$ represent the family of IVFs through $\beta$-levels. If $\Psi \in \mathcal{F} \mathcal{R}_{([\sqcap, \nu], \beta)}$ and $\nabla:[\sqcap, \nu] \rightarrow \mathbb{R}, \nabla(\ddagger) \geq 0$, symmetric with respect to $\frac{\square+v}{2}$, then

$\frac{1}{v-\sqcap}(\mathrm{FR}) \int_{\Pi}^{v} \Psi(\ddagger) \nabla(\ddagger) \mathrm{d} \ddagger$

$\preccurlyeq[\Psi(\sqcap) \tilde{+} \Psi(v)] \int_{0}^{1} h(\xi) \nabla((1-\xi) \sqcap+\xi \nu) \mathrm{d} \xi$.

Proof. Let $\Psi$ be a $h$-convex FIVF. Then, for each $\beta \in[0,1]$, we have

$$
\begin{gathered}
\Psi *(\xi \sqcap+(1-\xi) \nu, \beta) \nabla(\xi \sqcap+(1-\xi) \nu) \\
\leq(h(\xi) \Psi *(\sqcap, \beta)+h(1-\xi) \Psi *(\nu, \beta)) \nabla\left(\begin{array}{c}
\xi u+ \\
(1-\xi) \nu
\end{array}\right), \\
\Psi^{*}(\xi \sqcap+(1-\xi) \nu, \beta) \nabla(\xi \sqcap+(1-\xi) \nu) \\
\leq\left(h(\xi) \Psi^{*}(\sqcap, \beta)+h(1-\xi) \Psi^{*}(\nu, \beta)\right) \nabla\left(\begin{array}{c}
\xi u+ \\
(1-\xi) \nu
\end{array}\right) .
\end{gathered}
$$

And 


$$
\begin{gathered}
\Psi *((1-\xi) \sqcap+\xi \nu, \beta) \nabla((1-\xi) \sqcap+\xi \nu) \\
\leq(h(1-\xi) \Psi *(\sqcap, \beta)+h(\xi) \Psi *(\nu, \beta)) \nabla\left(\begin{array}{c}
(1-\xi) u \\
+\xi \nu
\end{array}\right), \\
\Psi^{*}((1-\xi) \sqcap+\xi \nu, \beta) \nabla((1-\xi) \sqcap+\xi v) \\
\leq\left(h(1-\xi) \Psi^{*}(\sqcap, \beta)+h(\xi) \Psi^{*}(\nu, \beta)\right) \nabla\left(\begin{array}{c}
(1-\xi) u \\
+\xi \nu
\end{array}\right) .
\end{gathered}
$$

After adding (21) and (22), and integrating over [0,1], we get

$$
\begin{aligned}
& \int_{0}^{1} \Psi *(\xi \sqcap+(1-\xi) \nu, \beta) \nabla(\xi \sqcap+(1-\xi) v) d \xi \\
& +\int_{0}^{1} \Psi *((1-\xi) \sqcap+\xi \nu, \beta) \nabla((1-\xi) \sqcap+\xi \nu) d \xi \\
& \leq \int_{0}^{1}\left[\begin{array}{c}
\Psi *(\sqcap, \beta)\left\{\begin{array}{c}
h(\xi) \nabla(\xi \sqcap+(1-\xi) \nu) \\
+h(1-\xi) \nabla((1-\xi) \sqcap+\xi \nu)
\end{array}\right. \\
+\Psi *(\nu, \beta)\left\{\begin{array}{c}
h(1-\xi) \nabla(\xi \sqcap+(1-\xi) \nu) \\
+h(\xi) \nabla((1-\xi) \sqcap+\xi \nu)
\end{array}\right\}
\end{array}\right] d \xi, \\
& \int_{0}^{1} \Psi^{*}((1-\xi) \sqcap+\xi \nu, \beta) \nabla((1-\xi) \sqcap+\xi \nu) d \xi \\
& +\int_{0}^{1} \Psi^{*}(\xi \sqcap+(1-\xi) \nu, \beta) \nabla(\xi \sqcap+(1-\xi) v) d \xi \\
& \leq \int_{0}^{1}\left[\begin{array}{c}
\Psi^{*}(\sqcap, \beta)\left\{\begin{array}{c}
h(\xi) \nabla(\xi \sqcap+(1-\xi) \nu) \\
+h(1-\xi) \nabla((1-\xi) \sqcap+\xi \nu)
\end{array}\right\} \\
+\Psi^{*}(\nu, \beta)\left\{\begin{array}{c}
h(1-\xi) \nabla(\xi \sqcap+(1-\xi) \nu) \\
+h(\xi) \nabla((1-\xi) \sqcap+\xi \nu)
\end{array}\right\}
\end{array}\right] d \xi . \\
& =2 \Psi *(\sqcap, \beta) \int_{0}^{1} h(\xi) \nabla(\xi \sqcap+(1-\xi) v) d \xi \\
& +2 \Psi *(\nu, \beta) \int_{0}^{1} h(\xi) \nabla((1-\xi) \sqcap+\xi \nu) d \xi, \\
& =2 \Psi^{*}(\sqcap, \beta) \int_{0}^{1} h(\xi) \nabla(\xi \sqcap+(1-\xi) v) d \xi \\
& +2 \Psi^{*}(\nu, \beta) \int_{0}^{1} h(\xi) \nabla((1-\xi) \sqcap+\xi \nu) d \xi .
\end{aligned}
$$

Since $\nabla$ is symmetric, then

$$
\begin{aligned}
& =2[\Psi *(\sqcap, \beta)+\Psi *(\nu, \beta)] \int_{0}^{1} h(\xi) \nabla\left(\begin{array}{c}
(1-\xi) u \\
+\xi \nu
\end{array}\right) d \xi, \\
& =2\left[\Psi^{*}(\sqcap, \beta)+\Psi^{*}(\nu, \beta)\right] \int_{0}^{1} h(\xi) \nabla\left(\begin{array}{c}
(1-\xi) u \\
+\xi \nu
\end{array}\right) d \xi .
\end{aligned}
$$

Since

$\int_{0}^{1} \Psi *(\xi \sqcap+(1-\xi) \nu, \beta) \nabla(\xi \sqcap+(1-\xi) \nu) d \xi$

$=\int_{0}^{1} \Psi *((1-\xi) \sqcap+\xi \nu, \beta) \nabla((1-\xi) \sqcap+\xi \nu) d \xi$

$$
=\frac{1}{v-\sqcap} \int_{\Pi}^{v} \Psi *(\ddagger, \beta) \nabla(\ddagger) \mathrm{d} \ddagger
$$

$\int_{0}^{1} \Psi^{*}((1-\xi) \sqcap+\xi \nu, \beta) \nabla((1-\xi) \sqcap+\xi \nu) d \xi$

$=\int_{0}^{1} \Psi^{*}(\xi \sqcap+(1-\xi) \nu, \beta) \nabla(\xi \sqcap+(1-\xi) \nu) d \xi$

$$
=\frac{1}{v-\sqcap} \int_{\Gamma}^{v} \Psi^{*}(\ddagger, \beta) \nabla(\ddagger) \mathrm{d} \ddagger \text {. }
$$

From (23) and (24), we have

$$
\begin{gathered}
\frac{1}{v-\sqcap} \int_{\Pi}^{\nu} \Psi *(\ddagger, \beta) \nabla(\ddagger) \mathrm{d} \ddagger \\
\leq[\Psi *(\sqcap, \beta)+\Psi *(\nu, \beta)] \int_{0}^{1} h(\xi) \nabla((1-\xi) \sqcap+\xi \nu) d \xi, \\
\quad \frac{1}{v-\sqcap} \int_{\Pi}^{v} \Psi^{*}(\ddagger, \beta) \nabla(\ddagger) \mathrm{d} \ddagger \\
\leq\left[\Psi^{*}(\sqcap, \beta)+\Psi^{*}(\nu, \beta)\right] \int_{0}^{1} h(\xi) \nabla((1-\xi) \sqcap+\xi \nu) d \xi,
\end{gathered}
$$

that is

$$
\begin{aligned}
& {\left[\frac{1}{v-\sqcap} \int_{\sqcap}^{v} \Psi *(\ddagger, \beta) \nabla(\ddagger) d \ddagger, \frac{1}{v-\sqcap} \int_{\Pi}^{v} \Psi^{*}(\ddagger, \beta) \nabla(\ddagger) \mathrm{d} \ddagger\right]} \\
& \leq_{l}\left[\Psi *(\sqcap, \beta)+\Psi *(\nu, \beta), \Psi^{*}(\sqcap, \beta)+\Psi^{*}(\nu, \beta)\right] \\
& \quad \int_{0}^{1} h(\xi) \nabla((1-\xi) \sqcap+\xi v) \mathrm{d} \xi
\end{aligned}
$$

hence

$$
\begin{aligned}
& \frac{1}{v-\sqcap}(\mathrm{FR}) \int_{\sqcap}^{v} \Psi(\ddagger) \nabla(\ddagger) \mathrm{d} \ddagger \\
& \leqslant[\Psi(\sqcap) \tilde{+} \Psi(\nu)] \int_{0}^{1} h(\xi) \nabla((1-\xi) \sqcap+\xi \nu) \mathrm{d} \xi,
\end{aligned}
$$

this concludes the proof.

Next, we construct first $H H$-Fej'er inequality for $h$-convex FIVF, which generalizes first $\mathrm{HH}$-Fej'er inequality for $h$-convex function, see [29].

Theorem 3.6 Let $\Psi:[\sqcap, v] \rightarrow \mathbb{F}_{C}(\mathbb{R})$ be a h-convex FIVF with $\Pi<v$ and $h:[0,1] \rightarrow \mathbb{R}^{+}$, and for all $\beta \in[0,1], \quad \Psi_{\beta}: K \subset \mathbb{R} \rightarrow \mathcal{K}_{C}{ }^{+} \subset \mathcal{K}_{C}$ represent the family of IVFs through $\beta$-levels. If $\Psi \in \mathcal{F R}_{([\sqcap, \nu], \beta)}$ and $\nabla:[\sqcap, v] \rightarrow \mathbb{R}, \nabla(\ddagger) \geq 0$, symmetric with respect to $\frac{\square+v}{2}$, and $\int_{\Gamma}^{v} \nabla(\ddagger) \mathrm{d} \ddagger>0$, then

$\Psi\left(\frac{\sqcap+v}{2}\right) \leqslant \frac{2 h\left(\frac{1}{2}\right)}{\int_{\Pi}^{v} \nabla(\ddagger) d \ddagger}(\mathrm{FR}) \int_{\sqcap}^{v} \Psi(\ddagger) \nabla(\ddagger) \mathrm{d} \ddagger$.

Proof. Since $\Psi$ is a $h$-convex, then for $\beta \in[0,1]$, we have

$$
\begin{gathered}
\Psi *\left(\frac{\square+v}{2}, \beta\right) \\
\leq h\left(\frac{1}{2}\right)\left(\begin{array}{c}
\Psi *(\xi \sqcap+(1-\xi) \nu, \beta) \\
+\Psi *((1-\xi) \sqcap+\xi \nu, \beta)
\end{array}\right), \\
\Psi^{*}\left(\frac{\square+v}{2}, \beta\right) \\
\leq h\left(\frac{1}{2}\right)\left(\begin{array}{c}
\Psi^{*}(\xi \sqcap+(1-\xi) \nu, \beta) \\
+\Psi^{*}((1-\xi) \sqcap+\xi \nu, \beta)
\end{array}\right),
\end{gathered}
$$

B y

$$
\text { m u } 1 \mathrm{t} \text { i p } 1 \text { y i n g }
$$

( 26 )

b y $\nabla(\xi \sqcap+(1-\xi) \nu)=\nabla((1-\xi) \sqcap+\xi \nu)$ and integrate it by $\xi$ over $[0,1]$, we obtain 


$$
\begin{gathered}
\Psi *\left(\frac{\sqcap+\nu}{2}, \beta\right) \int_{0}^{1} \nabla((1-\xi) \sqcap+\xi \nu) \mathrm{d} \xi \\
\leq h\left(\frac{1}{2}\right)\left(\begin{array}{c}
\int_{0}^{1} \Psi *(\xi \sqcap+(1-\xi) \nu, \beta) \nabla\left(\begin{array}{c}
\xi u+ \\
(1-\xi) \nu
\end{array}\right) \mathrm{d} \xi \\
+\int_{0}^{1} \Psi *((1-\xi) \sqcap+\xi \nu, \beta) \nabla\left(\begin{array}{c}
(1-\xi) u \\
+\xi \nu
\end{array}\right) \mathrm{d} \xi
\end{array}\right) \\
\Psi^{*}\left(\frac{\square+v}{2}, \beta\right) \int_{0}^{1} \nabla((1-\xi) \sqcap+\xi \nu) \mathrm{d} \xi \\
\leq h\left(\frac{1}{2}\right)\left(\begin{array}{c}
\int_{0}^{1} \Psi^{*}(\xi \sqcap+(1-\xi) \nu, \beta) \nabla\left(\begin{array}{c}
\xi u+ \\
(1-\xi) \nu
\end{array}\right) \mathrm{d} \xi \\
+{ }_{0}^{1} \Psi^{*}((1-\xi) \sqcap+\xi \nu, \beta) \nabla\left(\begin{array}{c}
(1-\xi) u \\
+\xi \nu
\end{array}\right) \mathrm{d} \xi
\end{array}\right)
\end{gathered}
$$

Since

$\int_{0}^{1} \Psi *(\xi \sqcap+(1-\xi) v, \beta) \nabla(\xi \sqcap+(1-\xi) v) d \xi$

$=\int_{0}^{1} \Psi *((1-\xi) \sqcap+\xi \nu, \beta) \nabla((1-\xi) \sqcap+\xi \nu) d \xi$, $=\frac{1}{v-\Pi} \int_{\Pi}^{v} \Psi *(\ddagger, \beta) \nabla(\ddagger) \mathrm{d} \ddagger$,

$\int_{0}^{1} \Psi^{*}((1-\xi) \sqcap+\xi \nu, \beta) \nabla((1-\xi) \sqcap+\xi v) d \xi$

$=\int_{0}^{1} \Psi^{*}(\xi \sqcap+(1-\xi) v, \beta) \nabla(\xi \sqcap+(1-\xi) v) d \xi$,

$$
=\frac{1}{v-\sqcap} \int_{\Pi}^{v} \Psi^{*}(\ddagger, \beta) \nabla(\ddagger) \mathrm{d} \ddagger,
$$

From (27) and (28), we have

$\Psi_{*}\left(\frac{\mu+v}{2}, \beta\right) \leq \frac{2 h\left(\frac{1}{2}\right)}{\int_{\mu}^{v} \nabla(z) \mathrm{d} z} \int_{\mu}^{v} \Psi_{*}(z, \beta) \nabla(z) \mathrm{d} z$,

$\Psi^{*}\left(\frac{\mu+v}{2}, \beta\right) \leq \frac{2 h\left(\frac{1}{2}\right)}{\int_{\mu}^{v} \nabla(z) \mathrm{d} z} \int_{\mu}^{\nu} \Psi^{*}(z, \beta) \nabla(z) \mathrm{d} z$.

From which, we have

$\left[\Psi_{*}\left(\frac{\mu+v}{2}, \beta\right), \Psi^{*}\left(\frac{\mu+v}{2}, \beta\right)\right]$
$\leq \frac{2 h\left(\frac{1}{2}\right)}{l \int_{\mu}^{v} \nabla(z) \mathrm{d} z}\left[\int_{\mu}^{v} \Psi_{*}(z, \beta) \nabla(z) \mathrm{d} z, \int_{\mu}^{v} \Psi^{*}(z, \beta) \nabla(z) \mathrm{d} z\right]$,

that is

$\Psi\left(\frac{\mu+v}{2}\right) \leqslant \frac{2 h\left(\frac{1}{2}\right)}{\int_{\mu}^{v} \nabla(z) \mathrm{d} z}(\mathrm{FR}) \int_{\mu}^{v} \Psi(z) \nabla(z) \mathrm{d} z$,

Then we complete the proof.

Remark 3.2. If $h(\xi)=\xi$, then inequalities in Theorem 10 and 11 reduces for convex FIVFs which are also new one.

If $\Psi_{*}(z, \beta)=\Psi^{*}(z, \beta)$ with $\beta=1$ and $h(\xi)=\xi$, then Theorem 10 and 11 reduce to classical first and second $H H$-Fejér inequality for convex function, see [31].

Example 3.5. We consider $h(\xi)=\xi$ and the FIVF $\Psi:[1,4] \rightarrow \mathbb{F}_{C}(\mathbb{R})$ defined by,
$\Psi(z)(\sigma)=\left\{\begin{array}{c}\frac{\sigma-e^{z}}{4 e^{e^{z}}-\sigma}, \sigma \in\left[e^{z}, 2 e^{z}\right], \\ \frac{2 e^{z}}{0}, \sigma \in\left(2 e^{z}, 4 e^{z}\right], \\ 0, \text { otherwise. }\end{array}\right.$

Then, for each $\beta \in[0,1], \quad$ we have $\Psi_{\beta}(z)=\left[(1+\beta) e^{z}, 2(2-\beta) e^{z}\right]$. Since end point functions $\Psi_{*}(z, \beta), \Psi^{*}(z, \beta)$ are $h$-convex functions, for each $\beta \in[0,1]$, then $\Psi(z)$ is $h$-convex FIVF. If

$\nabla(z)=\left\{\begin{array}{l}z-1, \sigma \in\left[1, \frac{5}{2}\right], \\ 4-z, \sigma \in\left(\frac{5}{2}, 4\right] .\end{array}\right.$

Then, we have

$\frac{1}{3} \int_{1}^{4} \Psi_{*}(z, \beta) \nabla(z) \mathrm{d} z=\frac{1}{3} \int_{1}^{4} \Psi_{*}(z, \beta) \nabla(z) \mathrm{d} z$

$=\frac{1}{3} \int_{1}^{\frac{5}{2}} \Psi_{*}(z, \beta) \nabla(z) \mathrm{d} z+\frac{1}{3} \int_{\frac{5}{2}}^{4} \Psi_{*}(z, \beta) \nabla(z) \mathrm{d} z$,

$\frac{1}{3} \int_{1}^{4} \Psi^{*}(z, \beta) \nabla(z) \mathrm{d} z=\frac{1}{3} \int_{1}^{4} \Psi^{*}(z, \beta) \nabla(z) \mathrm{d} z$

$=\frac{1}{3} \int_{1}^{\frac{5}{2}} \Psi^{*}(z, \beta) \nabla(z) \mathrm{d} z+\frac{1}{3} \int_{\frac{5}{2}}^{4} \Psi^{*}(z, \beta) \nabla(z) \mathrm{d} z$,

$=\frac{1}{3}(1+\beta) \int_{1}^{\frac{5}{2}} e^{z}(z-1) \mathrm{d} z+\frac{1}{3}(1+\beta) \int_{\frac{5}{2}}^{4} e^{z}(4-z) \mathrm{d} z$

$$
\approx 11(1+\beta)
$$

$=\frac{2}{3}(2-\beta) \int_{1}^{\frac{5}{2}} e^{z}(z-1) \mathrm{d} z+\frac{2}{3}(2-\beta) \int_{\frac{5}{2}}^{4} e^{z}(4-z) \mathrm{d} z$

$$
\approx 22(2-\beta)
$$

and

$$
\left[\Psi_{*}(\mu, \beta)+\Psi_{*}(\nu, \beta)\right] \int_{0}^{1} \xi \nabla((1-\xi) \mu+\xi \nu) d \xi
$$

$\left[\Psi^{*}(\mu, \beta)+\Psi^{*}(\nu, \beta)\right] \int_{0}^{1} \xi \nabla((1-\xi) \mu+\xi \nu) d \xi$

$$
\begin{gathered}
=(1+\beta)\left[e+e^{4}\right]\left[\int_{0}^{\frac{1}{2}} 3 \xi^{2} \mathrm{~d} z+\int_{\frac{1}{2}}^{1} \xi(3-3 \xi) \mathrm{d} \xi\right] \\
\approx \int^{\frac{43}{2}}(1+\beta) \\
=2(2-\beta)\left[e+e^{4}\right]\left[\int_{0}^{\frac{1}{2}} 3 \xi^{2} \mathrm{~d} z+\int_{\frac{1}{2}}^{1} \xi(3-3 \xi) \mathrm{d} \xi\right] \\
\approx 43(2-\beta) .
\end{gathered}
$$


From (31) and (30), we have

$[11(1+\beta), 22(2-\beta)] \leq_{I}\left[\frac{43}{2}(1+\beta), 43(2-\beta)\right]$,

for each $\beta \in[0,1]$. Hence, Theorem 3.5 is verified.

For Theorem 3.6, we have

$\Psi_{*}\left(\frac{\mu+v}{2}, \beta\right) \approx \frac{61}{5}(1+\beta)$,

$\Psi *\left(\frac{\mu+v}{2}, \beta\right) \approx \frac{122}{5}(2-\beta)$,

$\int_{\mu}^{\nu} \nabla(z) d z=\int_{1}^{\frac{5}{2}}(z-1) \mathrm{d} z \int_{\frac{5}{2}}^{4}(4-z) \mathrm{d} z=\frac{9}{4}$

$\frac{1}{\int_{\mu}^{v} \nabla(z) \mathrm{d} z} \int_{1}^{4} \Psi_{*}(z, \beta) \nabla(z) \mathrm{d} z \approx \frac{73}{5}(1+\beta)$

$\frac{1}{\int_{\mu}^{v} \nabla(z) d z} \int_{1}^{4} \Psi^{*}(z, \beta) \nabla(z) \mathrm{d} z \approx \frac{293}{10}(2-\beta)$

From (31) and (32), we have

$\left[\frac{61}{5}(1+\beta), 24.4(2-\beta)\right] \leq{ }_{I}\left[\frac{73}{5}(1+\beta), \frac{293}{10}(2-\beta)\right]$.

Hence, Theorem 11 is verified.

\section{Conclusion}

This study introduced the class of $h$-convex FIVFs and established some new $\mathrm{H}-\mathrm{H}$ inequalities by means of fuzzy order relation on fuzzy-interval space. Moreover, we established strong relationship between $\mathrm{H}-\mathrm{H}-\mathrm{Fej}^{\prime}$ er type inequality and $h$-convex FIVF. We provided relevant examples to demonstrate the application of the theory produced in this research. To construct fuzzy-interval inequalities of FIVFs, we plan to use a variety of convex FIVFs. We hope that this notion will assist other authors in remunerating their contributions in other sectors of knowledge. In future, we try to explore this concept using different fuzzy fractional integral operators.

Acknowledgements The authors would like to thank the Rector, COMSATS University Islamabad, Islamabad, Pakistan, for providing excellent research and academic environment. This research has been partially supported by Ministerio de Ciencia, Innovaciony Universidades grant number PGC2018-0971-B-100 and Fundacion Seneca de la Region de Murcia Grant number 20783/PI/18

Author Contributions All authors contributed equally to the writing of this paper. All authors read and approved the final manuscript.

Funding Not applicable.

\section{Declarations}

Conflict of interest The authors declare that they have no competing interests.

Open Access This article is licensed under a Creative Commons Attribution 4.0 International License, which permits use, sharing, adaptation, distribution and reproduction in any medium or format, as long as you give appropriate credit to the original author(s) and the source, provide a link to the Creative Commons licence, and indicate if changes were made. The images or other third party material in this article are included in the article's Creative Commons licence, unless indicated otherwise in a credit line to the material. If material is not included in the article's Creative Commons licence and your intended use is not permitted by statutory regulation or exceeds the permitted use, you will need to obtain permission directly from the copyright holder. To view a copy of this licence, visit http://creativecommons.org/licenses/by/4.0/.

\section{References}

1. Alomari, M., Darus, M., Dragomir, S.S., Cerone, P.: Ostrowski type inequalities for functions whose derivatives are $s$-convex in the second sense. Appl. Math. Lett. 23(9), 1071-1076 (2010)

2. Anderson, G.D., Vamanamurthy, M.K., Vuorinen, M.: Generalized convexity and inequalities. J. Math. Anal. Appl. 335(2), 1294-1308 (2007)

3. Avci, M., Kavurmaci, H., Ozdemir, M.E.: New inequalities of Hermite-Hadamard type via $s$-convex functions in the second sense with applications. Appl. Math. Comput. 217(12), 5171$5176(2011)$

4. Awan, M.U., Noor, M.A., Noor, K.I.: Hermite-Hadamard inequalities for exponentially convex functions. Appl. Math. Inform. Sci. 12(2), 405-409 (2018)

5. Bede, B.: Studies in Fuzziness and Soft Computing. In: Mathematics of Fuzzy Sets and Fuzzy Logic, vol. 295, Springer (2013)

6. Breckner, W.W.: Stetigkeitsaussagen für eine Klasse verallgemeinerter konvexer funktionen in topologischen linearen Räumen. Appl. Math. Comput. 23(37), 13-20 (1978)

7. Chen, F., Wu, S.: Integral inequalities of Hermite-Hadamard type for products of two $h$-convex functions. Abstr. Appl. Anal. 6, 1-6 (2014)

8. Chalco-Cano, Y., Flores-Franuli`c, A., Román-Flores, H.: Ostrowski type inequalities for interval-valued functions using generalized Hukuhara derivative. Comput. Appl. Math. 31(3), 457-472 (2012)

9. Chalco-Cano, Y., Lodwick, W.A., Condori-Equice, W.: Ostrowski type inequalities and applications in numerical integration for interval-valued functions. Soft. Comput. 19(11), 3293-3300 (2015)

10. Costa, T.M., Roman-Flores, H.: Some integral inequalities for fuzzy-interval-valued functions. Inf. Sci. 420, 110-125 (2017)

11. Costa, T.M.: Jensen's inequality type integral for fuzzy-intervalvalued functions. Fuzzy Sets Syst. 327, 31-47 (2017)

12. Costa, T.M., Román-Flores, H., Chalco-Cano, Y.: Opial-type inequalities for interval-valued functions. Fuzzy Sets Syst. 358, 48-63 (2019)

13. Diamond, P., Kloeden, P.E.: Metric Spaces of Fuzzy Sets: Theory and Applications. World Scientific (1994)

14. Dragomir, S.S., Pecaric, J., Persson, L.E.: Some inequalities of Hadamard type. Soochow J. Math 21(3), 335-341 (1995)

15. Goetschel, R., Jr., Voxman, W.: Elementary fuzzy calculus. Fuzzy Sets Syst. 18(1), 31-43 (1986) 
16. Hadamard, J.: Étude sur les propriétés des fonctions entières et en particulier d'une fonction considérée par Riemann. Journal De MathématiquesPpures Et Appliquées 5, 171-216 (1893)

17. Hermite, C.: Sur deux limites d'une intégrale définie. Mathesis 3(1), 1-82 (1883)

18. Hudzik, H., Maligranda, L.: Some remarks on $s$-convex functions. Aequationes Math. 48, 100-111 (1994)

19. Iscan, I.: A new generalization of some integral inequalities for $(\alpha, \mathrm{m})$-convex functions. Math. Sci 7(1), 1-8 (2013)

20. Iscan, I.: Hermite-Hadamard type inequalities for harmonically convex functions. Hacettepe J. Math. Stat. 43, 935-942 (2013)

21. Iscan, I.: Hermite-Hadamard type inequalities for $p$-convex functions. Int. J. Anal. Appl. 11, 137-145 (2016)

22. Kaleva, O.: Fuzzy differential equations. Fuzzy Sets Syst. 24(3), 301-317 (1987)

23. Kulish, U., Miranker, W.: Computer Arithmetic in Theory and Practice. Academic Press, New York (2014)

24. Moore, R.E.: Interval Analysis. Prentice Hall, Englewood Cliffs (1966)

25. Nanda, S., Kar, K.: Convex fuzzy mappings. Fuzzy Sets Syst. 48(1), 129-132 (1992)

26. Osuna-G'omez, R., Jimenez-Gamero, M. D., Chalco-Cano, Y., Rojas-Medar M. A.: Hadamard and Jensen Inequalities for s-Convex Fuzzy Processes. In: Soft Methodology and Random Information Systems. Advances in Soft Computing, vol. 126. Springer, Berlin, Heidelberg, pp. 1-15 (2004)

27. Puri, M.L., Ralescu, D.A.: Fuzzy random variables. Read. Fuzzy Sets Intell. Syst. 114, 409-422 (1986)

28. Román-Flores, H., Chalco-Cano, Y., Lodwick, W.A.: Some integral inequalities for interval-valued functions. Comput. Appl. Math. 37(2), 1306-1318 (2018)

29. Varošanec, S.: On h-convexity. J. Math. Anal. Appl. 326(1), 303311 (2007)

30. Zhao, D.F., An, T.Q., Ye, G.J., Liu, W.: New Jensen and HermiteHadamard type inequalities for h-convex interval-valued functions. J. Inequal. Appl. 2018(1), 1-14 (2018)

31. Fej'er, L.: Uberdie Fourierreihen II, Math. Naturwise. Anz Ungar Akad Wiss. 1906(24): 369-390

32. Khan, M.B., Noor, M.A., Noor, K.I., Chu, Y.-M.: New HermiteHadamard type inequalities for $(\mathrm{h} 1, \mathrm{~h} 2)$-convex fuzzy-intervalvalued functions. Adv. Differ. Equ. 2021, 6-20 (2021)

33. Liu, P., Khan, M.B., Noor, M.A., Noor, K.I.: New Hermite-Hadamard and Jensen inequalities for log-s-convex fuzzy-intervalvalued functions in the second sense. Complex Intell. Syst. 2021, $1-15(2021)$

34. Khan, M.B., Noor, M.A., Abdullah, L., Chu, Y.M.: Some new classes of preinvex fuzzy-interval-valued functions and inequalities. Int. J. Comput. Intell. Syst. 14(1), 1403-1418 (2021)

35. Khan, M.B., Mohammed, P.O., Noor, M.A., Hamed, Y.S.: New Hermite-Hadamard inequalities in fuzzy-interval fractional calculus and related inequalities. Symmetry 13(4), 673 (2021)
36. Liu, P., Khan, M.B., Noor, M.A., Noor, K.I.: On strongly generalized preinvex fuzzy mappings. J. Math. (2021). https://doi.org/10. $1155 / 2021 / 6657602$

37. Khan, M.B., Noor, M.A., Noor, K.I., Ab Ghani, A.T., Abdullah, L.: Extended perturbed mixed variational-like inequalities for fuzzy mappings. J. Math. (2021). https://doi.org/10.1155/2021/ 6652930

38. Khan, M.B., Mohammed, P.O., Noor, M.A., Alsharif, A.M., Noor, K.I.: New fuzzy-interval inequalities in fuzzy-interval fractional calculus by means of fuzzy order relation. AIMS Math. 6, 10964 10988 (2021)

39. Arqub, O.A., Al-Smadi, M.: Fuzzy conformable fractional differential equations: novel extended approach and new numerical solutions. Soft. Comput. 2020, 1-22 (2020)

40. Arqub, O.A.: Adaptation of reproducing kernel algorithm for solving fuzzy Fredholm-Volterra integrodifferential equations. Neural Comput. Appl. 28(7), 1591-1610 (2017)

41. Arqub, O.A., Al-Smadi, M., Momani, S., Hayat, T.: Application of reproducing kernel algorithm for solving second-order, two-point fuzzy boundary value problems. Soft. Comput. 21(23), 7191-7206 (2017)

42. Ata, E., Kiymaz, I.O.: A study on certain properties of generalized special functions defined by Fox-Wright function. Appl. Math. Nonlinear Sci. 5, 147-162 (2020)

43. Ilhan, E., Kiymaz, I.O.: A generalization of truncated M-fractional derivative and applications to fractional differential equations. Appl. Math. Nonlinear Sci. 5, 171-188 (2020)

44. Sahin, R., Yagci, O.: Fractional calculus of the extended hypergeometric function. Appl. Math. Nonlinear Sci. 5, 369-384 (2020)

45. Kaur, D., Agarwal, P., Rakshit, M., Chand, M.: Fractional calculus involving (p, q)-Mathieu type series. Appl. Math. Nonlinear Sci. 5, 15-34 (2020)

46. Kabra, S., Nagar, H., Nisar, K.S., Suthar, D.L.: The MarichevSaigo-Maeda fractional calculus operators pertaining to the generalized k-Struve function. Appl. Math. Nonlinear Sci. 5, 593-602 (2020)

47. Khan, M.B., Mohammed, P.O., Noor, M.A., Abualnaja, K.M.: Fuzzy integral inequalities on coordinates of convex fuzzy interval-valued functions. Math. Biosci. Eng. 18, 6552-6580 (2021)

48. Khan, M.B., Mohammed, P.O., Noor, Baleanu, D., Guirao, J.L.G.: Some New Fractional Estimates of Inequalities for LR-p-Convex Interval-Valued Functions by Means of Pseudo Order Relation. Axioms. 10(3), 175 (2021)

Publisher's Note Springer Nature remains neutral with regard to jurisdictional claims in published maps and institutional affiliations. 